Primordial black hole and wormhole formation by domain walls

To cite this article: Heling Deng et al JCAP04(2017)050

View the article online for updates and enhancements.

\section{Recent citations}

Gravitational decoupling and the PicardLefschetz approach

Jon Brown et al

Primordial black holes-perspectives in gravitational wave astronomy

Misao Sasaki et al

Consistent cosmic bubble embeddings S. Shajidul Haque and Bret Underwood 


\title{
Primordial black hole and wormhole formation by domain walls
}

\author{
Heling Deng, ${ }^{a}$ Jaume Garriga ${ }^{a, b}$ and Alexander Vilenkin ${ }^{a}$ \\ ${ }^{a}$ Institute of Cosmology, Tufts University, \\ 574 Boston Ave, Medford, MA, 02155 U.S.A. \\ ${ }^{b}$ Departament de Fisica Fonamental i Institut de Ciencies del Cosmos, \\ Universitat de Barcelona, Marti i Franques, 1, Barcelona, 08028 Spain \\ E-mail: heling.deng@tufts.edu, garriga@cosmos.phy.tufts.edu, \\ vilenkin@cosmos.phy.tufts.edu
}

Received December 23, 2016

Accepted March 21, 2017

Published April 28, 2017

\begin{abstract}
In theories with a broken discrete symmetry, Hubble sized spherical domain walls may spontaneously nucleate during inflation. These objects are subsequently stretched by the inflationary expansion, resulting in a broad distribution of sizes. The fate of the walls after inflation depends on their radius. Walls smaller than a critical radius fall within the cosmological horizon early on and collapse due to their own tension, forming ordinary black holes. But if a wall is large enough, its repulsive gravitational field becomes dominant much before the wall can fall within the cosmological horizon. In this "supercritical" case, a wormhole throat develops, connecting the ambient exterior FRW universe with an interior baby universe, where the exponential growth of the wall radius takes place. The wormhole pinches off in a time-scale comparable to its light-crossing time, and black holes are formed at its two mouths. As discussed in previous work, the resulting black hole population has a wide distribution of masses and can have significant astrophysical effects. The mechanism of black hole formation has been previously studied for a dust-dominated universe. Here we investigate the case of a radiation-dominated universe, which is more relevant cosmologically, by using numerical simulations in order to find the initial mass of a black hole as a function of the wall size at the end of inflation. For large supercritical domain walls, this mass nearly saturates the upper bound according to which the black hole cannot be larger than the cosmological horizon. We also find that the subsequent accretion of radiation satisfies a scaling relation, resulting in a mass increase by about a factor of 2 .
\end{abstract}

Keywords: Cosmic strings, domain walls, monopoles, primordial black holes, Wormholes

ArXiv ePrint: 1612.03753 


\section{Contents}

1 Introduction 1

2 Dynamics of spherical domain walls in an ambient FRW universe 4

2.1 Model and equations of motion 4

2.2 Initial conditions $\quad 6$

2.3 Boundary conditions 7

$\begin{array}{lll}2.4 & \text { Expansions and Misner-Sharp mass } & 7\end{array}$

3 Subcritical wall $\left(t_{\sigma} \gtrsim t_{H}\right) \quad 8$

3.1 Equation of motion $\quad 8$

$\begin{array}{lll}3.2 & \text { Initial } \mathrm{BH} & 9\end{array}$

3.3 Final BH 9

4 Supercritical wall $\left(t_{\sigma} \lesssim t_{H}\right) \quad 12$

$\begin{array}{lll}4.1 & \text { Spacetime structure } & 13\end{array}$

$\begin{array}{lll}4.2 & \text { Upper bound on the initial BH mass } & 16\end{array}$

$\begin{array}{lll}4.3 & \text { Inner } \mathrm{BH} & 17\end{array}$

$\begin{array}{lll}4.4 & \text { Outer } \mathrm{BH} & 17\end{array}$

$\begin{array}{llr}5 & \text { Fireball } & 19\end{array}$

6 Summary and conclusions $\quad 20$

A Collapse of subcritical walls $\quad 24$

\section{Introduction}

Quantum fluctuations of matter fields during the inflationary epoch may have seeded the large scale structure we observe in the universe. While this process is usually considered in the perturbative regime, it was recently pointed out in ref. [1] that non-perturbative quantum effects could also contribute to the formation of structure on astrophysically relevant scales. Here we will focus on the scenario where the spontaneous nucleation of spherical domain walls during inflation leads to a population of primordial black holes, with a wide spectrum of masses.

Domain walls are two dimensional topological defects that may arise in theories with a spontaneously broken discrete symmetry, separating neighboring regions where the field is in different vacua. They are characterized by a finite energy density $\sigma$ per unit surface, and a tension of equal magnitude. Such tension, or negative pressure, is the source of a repulsive gravitational field. In the thin wall limit, the metric for a planar domain wall in the $y z$-plane (in an otherwise empty space) is given by [2-4]

$$
d s^{2}=-\left(1-\frac{|x|}{t_{\sigma}}\right)^{2} d t^{2}+d x^{2}+\left(1-\frac{|x|}{t_{\sigma}}\right)^{2} e^{2 t / t_{\sigma}}\left(d y^{2}+d z^{2}\right),
$$


where

$$
t_{\sigma}=\frac{1}{2 \pi G \sigma} .
$$

Here, $G$ is Newton's constant. The $(x, t)$-part of the metric is a $(1+1)$-dimensional Rindler space, and a geodesic observer near $x=0$ will recede from the wall with acceleration $t_{\sigma}^{-1}$. Also, the hypersurface $x=0$, corresponding to the worldsheet of the wall, inflates at the constant rate $H_{\sigma}=t_{\sigma}^{-1}$.

Cosmologically, domain walls may form by the Kibble mechanism at a phase transition, when the universe goes from a high temperature symmetric phase to a broken phase at lower temperatures. In this case, the distribution of defects at the time of formation will include closed walls as well as infinite walls of quite irregular shapes [2]. After the phase transition, the number of walls stretching across a horizon region is at least of order one, and such defects would come to dominate over the radiation or matter density at a time of order $t_{\sigma}$. This leads to a domain wall problem unless $t_{\sigma} \gtrsim t_{0}$ where $t_{0}$ is the current age of the universe. This severely limits the tension to be rather low $\sigma \lesssim 1 / G t_{0} \sim(100 \mathrm{MeV})^{3}$, while an even stronger constraint, $\sigma \lesssim(1 \mathrm{MeV})^{3}$ follows from the observed isotropy of the CMB [5].

Here we will be interested in a very different production mechanism, where the domain wall problem does not arise, and the above constraints on $\sigma$ do not apply. The scenario of our interest is the spontaneous nucleation of domain walls during inflation [6]. This can be thought of as quantum process by which the radius of a closed wall tunnels from vanishing size to Hubble size. The walls thus created are subsequently stretched by the inflationary expansion, resulting in a broad distribution of sizes. In the semiclassical limit, they are expected to be nearly spherical, with a size distribution which at the end of inflation takes the form,

$$
d n \approx \lambda(R) \frac{d R}{R^{4}} .
$$

Here, $n$ is the number density of walls, and $\lambda$ is the nucleation rate in Hubble units during inflation. The nucleation rate has an exponential dependence on the action $S_{E}$ of the semiclassical tunneling path, $\lambda \propto e^{-S_{E}}$, where $S_{E} \approx 2 \pi^{2} \sigma H_{i}^{-3}$. Here $H_{i}$ is the inflationary Hubble rate. The radii $R$ are in the range $1 \lesssim H_{i} R \lesssim e^{N}$, where $N \sim 60$ is the number of e-folding of slow roll inflation within observationally accessible scales. For constant $\lambda$ the distribution (1.3) would be scale invariant, but we may expect some dependence of $\lambda$ on $R$, since the value of $H_{i}$, and perhaps also $\sigma$, will slowly vary during inflation.

The dynamics of spherical domain walls in vacuum has been thoroughly studied in the literature. Ref. [4] considered the evolution of a spherical domain wall in asymptotically flat space. In this case the interior metric is Minkowski and the exterior is Schwarzschild. It was shown that if the wall is initially larger than the Schwarzschild radius, then it always collapses to a black hole singularity. Refs. [7-10] considered the more general case of a false vacuum bubble surrounded by true vacuum, and studied the motion of the domain wall at the boundary of these two regions by matching an interior de Sitter with a Schwarzschild exterior metric. This includes, in particular, the case when the energy density inside the bubble vanishes, so that the only source of the gravitational field is the domain wall. A solution was found where a domain wall emerges from an initial "white hole" singularity, and expands without bound thereafter in a baby universe. The baby universe is initially connected to the asymptotically flat region by a wormhole, but the wormhole eventually pinches off, leading to a change in topology. Because of the initial white hole singularity, such solutions may be considered somewhat unphysical. However, as pointed out in ref. [1], a 
similar process can occur for sufficiently large walls in the aftermath of a standard inflationary phase. ${ }^{1}$ In this case there is no initial white hole singularity.

In our cosmological setting, the fate of the walls depends on their size $R$ at the time $t_{i}$ when inflation ends [1]. At that time, all walls have radii greater than the horizon and are essentially at rest with respect to the Hubble flow. A useful parameter is the time $t_{H}\left(r_{i}\right)$ when the comoving scale $r_{i}=R / a\left(t_{i}\right)$ would fall within the Hubble radius in an undisturbed FRW universe. In a dust or radiation-dominated universe, we have $t_{H}=8 r_{i}^{3} /\left(27 t_{i}^{2}\right)$, or $t_{H}=r_{i}^{2} /\left(4 t_{i}\right)$ respectively. Here, and for the rest of this paper, we will adopt the convention that $a\left(t_{i}\right)=1$, so that $r_{i}=R$.

A wall with $t_{H}\left(r_{i}\right) \ll t_{\sigma}$ is called "subcritical", and its gravitational field can be safely neglected before horizon crossing. The wall grows for a while due to the cosmological expansion, and at the time $t_{H}$ it realizes that it's actually a ball. Then it is forced by the surface tension to shrink to a size smaller than the corresponding Schwarzschild radius, forming an ordinary black hole. Afterwards, some of the nearby fluid falls into the black hole, leading to mass accretion.

On the other hand, a wall with $t_{H}\left(r_{i}\right) \gg t_{\sigma}$ is called "supercritical", and its gravitational effect becomes significant at time $t_{\sigma}$, before the wall has a chance to fall within the cosmological horizon. Due to its repulsive nature, the wall pushes fluid away, leaving two nearly empty layers in its vicinity, which separate the wall from an interior and an exterior FRW regions. The exterior FRW universe continues its power law Hubble expansion, but the wall radius grows exponentially in proper time. This is only possible because the wall creates a wormhole, through which it escapes into a baby universe. The wormhole pinches off in a timescale comparable to its light crossing time, and observers on either side of the wormhole throat will see a black hole forming, possibly with different masses on both sides.

The reason why there is no domain wall problem in this scenario can easily be understood as follows. Subcritical walls form black holes before their contribution to the energy (on the Hubble scale) can become significant. After that, they simply behave as a contribution to cold dark matter. On the other hand, for supercritical walls, the repulsive gravitational field becomes important at the time $t_{\sigma}$, when their energy density locally dominates over the matter density (in all Hubble patches containing a segment of the wall). As soon as this happens, the wall starts creating a wormhole, and the "domain wall problem" is exported into the resulting baby universe. All we are left with in the parent universe is a distribution of primordial black holes with a broad spectrum of masses [1].

The relative contribution of black holes of mass $M_{\mathrm{bh}}$ to the dark matter density $\rho_{m}$ is given by $f \equiv\left(M_{\mathrm{bh}} / \rho_{m}\right) d n\left(M_{\mathrm{bh}}\right) / d \log M_{\mathrm{bh}}$. This is determined by the initial distribution of wall sizes, eq. (1.3), by using the relation $M_{\mathrm{bh}}(R)$ which gives the black hole mass a function of the initial wall radius $R$. In ref. [14] this relation was studied numerically for subcritical walls in a dust-dominated background. In ref. [1] the limits of small and large walls were studied analytically for the case of a dust background. The limit of small walls in a radiation background was also discussed. Nonetheless, the more interesting case of large supercritical walls in a radiation-dominated universe was not analyzed quantitatively. In this case, pressure gradients create a flux of radiation across the wormhole, which affects the mass of the resulting black hole, but the impact of this effect is hard to estimate analytically.

In ref. [1], the mass of supercritical black holes was tentatively assumed to be of order $M_{\mathrm{bh}} \sim t_{H} / G$, saturating the condition that the apparent horizon of a black hole at the time

\footnotetext{
${ }^{1}$ The possibility of wormhole formation in cosmological spacetimes has also been discussed earlier in refs. [11-13], but without suggesting a realistic cosmological scenario where it can be realized.
} 
of formation cannot be bigger than the size of the cosmological horizon. This upper bound was indeed shown to correspond to the mass of large supercritical black holes in dust. For the case of a radiation, this relation was only suggested by the behavior of $M_{\mathrm{bh}}(R)$ for small walls, extrapolated up to the critical size. With this assumption, it was found that the peak of the distribution $f$ corresponds to black holes of mass $M_{\text {crit }} \sim t_{\sigma} / G$, coming from the collapse of a walls with $t_{H}\left(r_{i}\right) \sim t_{\sigma}$. In this paper, we will provide a more precise determination of $M_{\mathrm{bh}}(R)$, which should allow for a better comparison of the distribution $f$ with observations.

Since the nucleation rate $\lambda$ is highly suppressed for $\sigma \gg H_{i}^{3}$, and the semiclassical approximation breaks down for $\sigma \ll H_{i}^{3}$, this leaves $\sigma \sim H_{i}^{3}$ as the preferred range of parameters. For an inflationary Hubble rate in the interval $10^{14} \mathrm{GeV} \gtrsim H_{i} \gtrsim 100 \mathrm{GeV}$ the peak in $f$ lies within the range $10^{7} \mathrm{~kg} \lesssim M_{\text {crit }} \lesssim 10^{15} M_{\odot}$. This leaves ample room for potentially observable effects on astrophysical and cosmological scales. Black holes at the peak of the distribution would form well within the radiation-dominated era, and therefore it is important to explore this case in greater detail. This will be the subject of the present paper.

In section 2 we describe our model and the equations of motion. The masses of subcritical and supercritical black holes as a function of the initial radius are discussed in sections 3 and 4 , respectively. Section 5 is devoted to a description of the evolution of the interior of a supercritical wall filled with radiation. Section 6 summarizes our conclusions.

\section{Dynamics of spherical domain walls in an ambient FRW universe}

In this section we consider the equations of motion and boundary conditions for a spherical domain wall embedded in an otherwise homogeneous FRW universe, initially dominated by the energy density of a perfect fluid.

\subsection{Model and equations of motion}

We will be interested in a spherically symmetric situation, where the metric can be written in the form

$$
d s^{2}=-A(r, t) d t^{2}+B(r, t)^{2} d r^{2}+R(r, t)^{2} d \Omega^{2} .
$$

Here $R(r, t)$ is the area radius, which we assume to vanish at the origin of spherical coordinates, $R(0, t)=0$, and $d \Omega^{2} \equiv d \theta^{2}+\sin ^{2} \theta d \varphi^{2}$. There is some freedom in choosing the lapse function $A(r, t)$, and in what follows we set $A(r, t)=1$, which completely fixes the gauge. This is called geodesic slicing because observers at fixed spatial coordinates experience freefall [15].

A domain wall can be described by a scalar field $\phi(r, t)$ with a double-well self-interaction potential $V(\phi)$. The Lagrangian density is given by

$$
\mathcal{L}_{\phi}=\frac{1}{2}\left(\partial_{\alpha} \phi\right)\left(\partial^{\alpha} \phi\right)-V(\phi),
$$

and the corresponding energy-momentum tensor takes the form

$$
T_{\nu}^{(\phi) \mu}=\partial^{\mu} \phi \partial_{\nu} \phi-\delta_{\nu}^{\mu} \mathcal{L}_{\phi}
$$

The background in which the domain wall is embedded is initially dominated by a perfect fluid with energy-momentum tensor $T_{\nu}^{(f) \mu}=(\rho+p) u^{\mu} u_{\nu}+\delta_{\nu}^{\mu} p$, where $\rho(r, t)$ and $p(r, t)=w \rho(r, t)$ 
are respectively the energy density and pressure measured in the fluid frame ( $w=0$ for dust and $w=\frac{1}{3}$ for radiation). The fluid's 4-velocity can be written in the form

$$
u^{\mu}(r, t)=\left(\frac{1}{\sqrt{1-v^{2}}}, \frac{v}{B \sqrt{1-v^{2}}}, 0,0\right),
$$

where $v(r, t)$ is the fluid's 3-velocity relative to the comoving coordinate $r$. We shall assume that there is only gravitational interaction between the scalar field and the fluid, so that the total energy-momentum tensor is simply given by $T_{\nu}^{(\phi) \mu}+T_{\nu}^{(f) \mu}$.

Our goal is to solve the equations of motion in order to find $B(r, t), R(r, t), \phi(r, t)$, $\rho(r, t)$ and $v(r, t)$. Let $H_{i}$ be the Hubble parameter at the end of inflation. By the following replacements

$$
r \rightarrow \frac{r}{H_{i}}, \quad t \rightarrow \frac{t}{H_{i}}, \quad \rho \rightarrow M_{\mathrm{Pl}}^{2} H_{i}^{2} \rho, \quad \phi \rightarrow M_{\mathrm{Pl}} \phi, \quad V \rightarrow M_{\mathrm{Pl}}^{2} H_{i}^{2} V,
$$

where $M_{\mathrm{Pl}}=1 / \sqrt{G}$, all variables become dimensionless. For instance, $t_{H}$ becomes $2 r_{i}^{3} / 3$ for dust and $r_{i}^{2} / 2$ for radiation. In what follows we use the dimensionless variables.

Following $[16,17]$ we introduce

$$
U \equiv \dot{R}, \quad \Gamma \equiv \frac{R^{\prime}}{B}, \quad K \equiv \frac{\dot{B}}{B}+\frac{2 \dot{R}}{R},
$$

where ${ }^{\cdot} \equiv \partial / \partial t$ and ${ }^{\prime} \equiv \partial / \partial r$. The evolution equations then take the form

$$
\begin{aligned}
\dot{K} & =-\left(K-\frac{2 U}{R}\right)^{2}-2\left(\frac{U}{R}\right)^{2}-4 \pi\left(T_{00}+T_{1}^{1}+2 T_{2}^{2}\right) \\
\dot{U} & =-\frac{1-\Gamma^{2}+U^{2}}{2 R}-4 \pi R T_{1}^{1} \\
\dot{\Gamma} & =-\frac{4 \pi R T_{1}^{0}}{B} \\
\dot{\rho} & =\frac{(1+w) \rho}{1-w v^{2}}\left[v^{2}\left(K-\frac{2 U}{R}\right)-K-\frac{2 v \Gamma}{R}-\frac{v^{\prime}}{B}\right]-\frac{1-w}{1-w v^{2}} \frac{\rho^{\prime} v}{B} \\
\dot{v} & =\frac{\left(1-v^{2}\right)}{1-w v^{2}}\left[-v\left(K-\frac{2 U}{R}\right)+w v K+\frac{2 w v^{2} \Gamma}{R}-\frac{\left(1-v^{2}\right) w}{(1+w)} \frac{\rho^{\prime}}{\rho B}\right]-\frac{1-w}{1-w v^{2}} \frac{v^{\prime} v}{B} \\
\ddot{\phi} & =-K \dot{\phi}+\frac{1}{B R^{2}}\left(\frac{R^{2}}{B} \phi^{\prime}\right)^{\prime}-\partial_{\phi} V \\
\dot{B} & =B\left(K-\frac{2 U}{R}\right) \\
\dot{R} & =U
\end{aligned}
$$

where

$$
\begin{aligned}
T_{00} & =\frac{1+w v^{2}}{1-v^{2}} \rho+\frac{1}{2} \dot{\phi}^{2}+\frac{1}{2} \phi^{2}+V(\phi) \\
T_{1}^{1} & =\frac{w+v^{2}}{1-v^{2}} \rho+\frac{1}{2} \dot{\phi}^{2}+\frac{1}{2} \phi^{\prime 2}-V(\phi) \\
T_{2}^{2} & =w \rho+\frac{1}{2} \dot{\phi}^{2}-\frac{1}{2} \phi^{\prime 2}-V(\phi) \\
T_{1}^{0} & =\dot{\phi} \phi^{\prime}-\frac{1+w}{1-v^{2}} \rho v B .
\end{aligned}
$$


Eq. (2.7), (2.8) and (2.9) follow from Einstein equations, while eqs. (2.10) and (2.11) follow from the conservation of the fluid energy-momentum tensor, and eq. (2.12) is the scalar field equation. As for eqs. (2.13) and (2.14), they are satisfied by definition of $K$ and $U$ respectively. As we discuss below, we use a combination of the Hamiltonian and the momentum constraint in order to check for numerical accuracy (see eq. (2.27)).

A common technical difficulty in simulating a domain wall over a large dynamical range is that the spacetime around the wall is expanding. Hence, in comoving coordinates, the wall thickness is decreasing, and more precision is needed to describe its sharper profile. To deal with this problem, we adopt a conventional approach called adaptive mesh refinement (AMR) [18]. A detailed recent discussion of this scheme can be found, for example, in ref. [19]. The strategy is to assign a sufficient number of grid points to the wall as the comoving thickness decreases, while the grid density far away from the wall remains unaltered for the sake of simulation efficiency. We require at least $\sim 20$ grid points across the wall. To calculate the derivatives on such a non-uniform grid, we use the method given in ref. [20].

The difficulty becomes particularly acute when the wall starts inflating due to its selfgravity, since then $R$ grows exponentially in proper time, and the comoving thickness of the wall shrinks exponentially. As a result, mesh refinement becomes inefficient for supercritical walls after the time $t_{\sigma}$. We will deal with this issue in section 4 .

\section{$2.2 \quad$ Initial conditions}

For convenience, we choose the so-called sine-Gordon self-interaction potential for the scalar field,

$$
V(\phi)=\lambda \cos ^{2}\left(\frac{\phi}{2 \eta}-\frac{\pi}{2}\right) .
$$

The corresponding field equation has an analytic planar wall solution in flat spacetime, given by

$$
\phi_{w}(x)=-4 \eta \arctan \left(e^{\frac{x}{\eta} \sqrt{\frac{\lambda}{2}}}\right)+2 \eta \pi .
$$

We use this as the initial profile of the scalar field, in the form

$$
\phi\left(r, t_{i}\right)=\phi_{w}\left(r-r_{i}\right) .
$$

In dimensionless units (2.5), the initial energy density at the time $t_{i}$ in a FRW universe is given by $\rho_{i}=(3 / 8 \pi)$. The energy density in the core of the domain wall is assumed to be subdominant at that time, and so we require $\lambda \ll 1$. The thickness of the wall is given by $\delta \sim \eta / \sqrt{\lambda}$ and should be smaller than the initial Hubble radius, $\delta \lesssim 1$. It follows that we must choose $\eta \lesssim \sqrt{\lambda} \lesssim 1$. With this, the dimensionless surface tension $\sigma \sim \eta \sqrt{\lambda} \lesssim 1$ will also be small, and $t_{\sigma}=(2 \pi \sigma)^{-1} \gtrsim 1$. For supercritical walls we need $t_{\sigma} \lesssim t_{H}$. In particular, in the marginal case where $\sigma \sim 1$, all walls with initial size much larger than the Hubble radius, $r_{i} \gg 1$, will be supercritical.

For the metric components and fluid velocity we assume the initial conditions $B\left(r, t_{i}\right)=1$, $R\left(r, t_{i}\right)=r$ and $v\left(r, t_{i}\right)=0$. Further we assume that $\dot{\phi}\left(r, t_{i}\right)=0$, and that the small initial energy density of the wall is compensated for by a deficit in the energy density of the background fluid, so $\rho\left(r, t_{i}\right)=(3 / 8 \pi)-(1 / 2) \phi^{2}\left(r, t_{i}\right)-V(\phi)$. The initial values of $K$ and $U$ can be determined from $G_{00}=8 \pi G T_{00}$ and $G_{01}=8 \pi G T_{01}$, and we find $K\left(r, t_{i}\right)=3$ and $U\left(r, t_{i}\right)=r$, as in an FRW universe. 


\subsection{Boundary conditions}

We also need to set boundary conditions at $r=0$. Obviously $R(0, t)=0$ and $U(0, t)=0$. By regularity, $\rho^{\prime}(0, t)=0, v^{\prime}(0, t)=0, B^{\prime}(0, t)=0$ and $B(0, t)=\lim _{r \rightarrow 0} R(r, t) / r$, which gives $K(0, t)=\lim _{r \rightarrow 0} 3 U(r, t) / R(r, t)$ and $\Gamma(0, t)=1$.

Note that there are no physical restrictions at the outer boundary in our computational domain, because derivatives there do not necessarily vanish (as they do in an FRW region). However, the only physical perturbation comes from the rarefaction waves produced near the wall, and as long as we have a sufficiently large computational domain, we don't need to worry about reflected waves. Therefore, to evolve the outer boundary, we don't specify any boundary condition, but simply use one-sided derivatives [15]. This works well enough for our purposes.

\subsection{Expansions and Misner-Sharp mass}

Consider a spherical $r=$ const. surface and a congruence $k^{\mu}$ of null geodesics orthogonal to it, with expansion given by $\Theta \equiv \nabla_{\mu} k^{\mu}$. We denote by $\Theta_{\text {out }}$ and $\Theta_{\text {in }}$ the expansion of outgoing and ingoing radial null geodesics, respectively. A surface is trapped if both expansions are negative, and anti-trapped if both are positive. The expansion of null congruence is useful in characterizing black holes locally (see e.g. [15]). In our coordinate system, we have

$$
\Theta_{\text {out }} \propto \frac{U+\Gamma}{R}, \quad \Theta_{\text {in }} \propto \frac{U-\Gamma}{R} .
$$

In a flat FRW universe,

$$
\Theta_{\text {out }} \propto H+\frac{1}{R}, \quad \Theta_{\text {in }} \propto H-\frac{1}{R},
$$

which should be satisfied in regions far from the wall.

We may characterize the quasi-local mass of an object by adopting the concept of Misner-Sharp energy [21, 22], which for a general metric $d s^{2}=h_{a b} d x^{a} d x^{b}+R^{2} d \Omega^{2}$ is defined as

$$
M=\frac{R}{2}\left(1-h^{a b} \partial_{a} R \partial_{b} R\right),
$$

and in our coordinates is given by

$$
M=\frac{R}{2}\left(1-\Gamma^{2}+U^{2}\right) .
$$

For a perfect fluid, the energy-momentum tensor is diagonal. In this case,

$$
8 \pi T_{00}=G_{00}=\frac{2 M^{\prime}}{R^{2} R^{\prime}},
$$

which gives $M=4 \pi \int T_{00} R^{2} d R$. So it is natural to interpret $M$ as "mass". For an FRW universe, $M$ is the total mass of matter enclosed by a sphere with radius $R$. In the case of Schwarzschild spacetime, $M$ is the mass constant in the metric. However, in our case, $T_{\nu}^{\mu}$ is not diagonal due to the scalar field. Instead of eq. (2.26), now we have

$$
M^{\prime}=4 \pi R^{2}\left(R^{\prime} T_{00}-\dot{R} T_{01}\right) .
$$

We will use this equation as a check for numerical accuracy. 
An important point for us about the Misner-Sharp mass is that it is not necessarily monotonic in $r$, because $R$ is not necessarily monotonic in $r$. In supercritical cases, due to the existence of a wormhole, there is a local minimum of $R$ and the mass $M$ could decrease with increasing $r$.

The apparent horizon of a spherically symmetric black hole is a marginally trapped surface, where $\Theta_{\text {out }}=0$ and $\Theta_{\text {in }}<0$. By definition we have $R=2 M$ at the apparent horizon. We also define the white hole horizon and cosmological horizon to be a marginally anti-trapped hypersurface where $\Theta_{\text {in }}=0$ and $\Theta_{\text {out }}>0$. A spherical surface is anti-trapped (with $\Theta_{\text {in }}>0$ and $\Theta_{\text {out }}>0$ ) within the white hole horizon, and is normal (with $\Theta_{\text {in }}<0$ and $\Theta_{\text {out }}>0$ ) if it lies between the white hole horizon and the cosmological horizon. Note that by eq. (2.23), the cosmological horizon and the Hubble radius coincide in a flat FRW universe.

\section{Subcritical wall $\left(t_{\sigma} \gtrsim t_{H}\right)$}

\subsection{Equation of motion}

When $t_{\sigma} \gg t_{H}$, the wall's gravitational effect can be completely neglected until the wall is about to collapse. Hence, the dynamics is well approximated by that of a test wall moving in the FRW background. In this case the wall's equation of motion is given by [1]

$$
\ddot{r}+\left(4-3 a^{2} \dot{r}^{2}\right) H \dot{r}+\frac{2}{a^{2} r}\left(1-a^{2} \dot{r}^{2}\right)=0,
$$

where $r$ is the wall's comoving radius, $a$ is the scale factor, and $H \equiv \dot{a} / a$ is the Hubble parameter. When we get closer to the critical regime, the wall's gravitational effect should be taken into account, and a similar equation can be obtained (see appendix),

$$
\ddot{r}+\left(4-3 a^{2} \dot{r}^{2}\right) H \dot{r}+\frac{2}{a^{2} r}\left(1-a^{2} \dot{r}^{2}\right)=6 \pi \sigma \frac{\left(1-a^{2} \dot{r}^{2}\right)^{\frac{3}{2}}}{a} .
$$

This equation is derived by assuming that the FRW spacetime inside the wall is unperturbed. We see that the only difference between eq. (3.1) and eq. (3.2) is the right hand side, which is a term related to the surface tension. It is also shown in appendix that the time $t_{\mathrm{BH}}$ when the apparent horizon first appears is given by

$$
\left(H+\frac{1}{a r}\right) \sqrt{\frac{1+a \dot{r}}{1-a \dot{r}}}=4 \pi \sigma .
$$

It turns out eqs. (3.2) and (3.3) agree very well with our numerical solution of the full equations of motion.

For dust background they work even near the critical regime where $t_{\sigma} \sim t_{H}$ (see figure 1). In the case of radiation, the propagation of rarefaction waves produced near the wall influences the interior FRW region, but eq. (3.2) is satisfied to a large extent. Eqs. (3.2) and (3.3) can easily be solved numerically, and instead of running full simulations, it is much more convenient to use them to obtain the initial black hole mass. This is simply given by $M_{\mathrm{BHi}}=$ $(1 / 2) a\left(t_{\mathrm{BH}}\right) r\left(t_{\mathrm{BH}}\right)$, where $r\left(t_{\mathrm{BH}}\right)$ is the location where the apparent horizon first forms. 

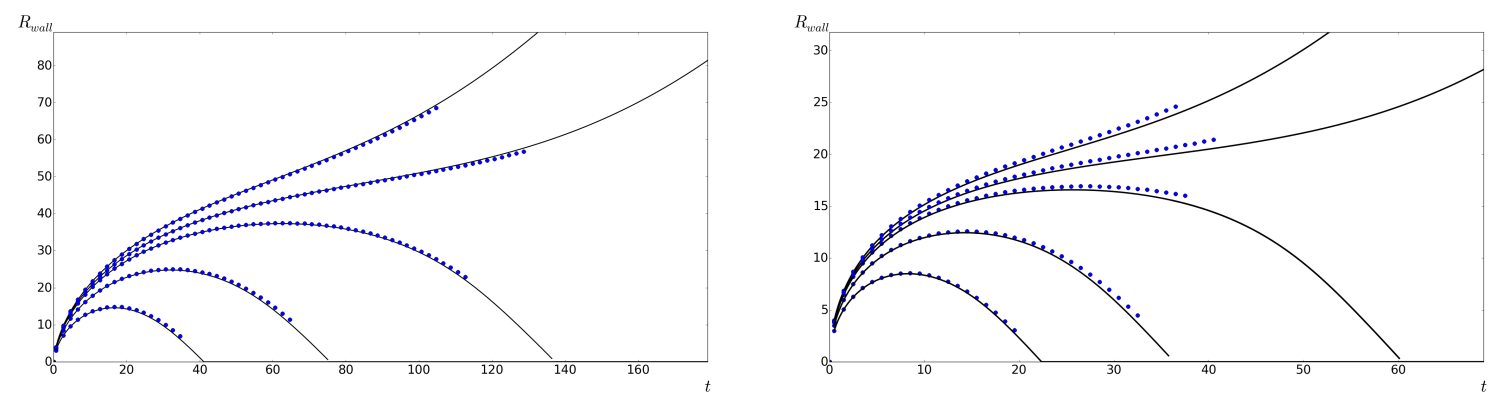

Figure 1. Trajectories of five walls in dust background (left panel, with $t_{\sigma} \approx 74$ ) and radiation background (right panel, with $t_{\sigma} \approx 30$ ), with $r_{i}=3,3.5,3.8,3.9,4$ from the bottom up. $R_{\text {wall }}=a r$ is the wall's area radius. Solid curves are obtained from eq. (3.2), and dots are from simulations. We can see that eq. (3.2) works very well in the case of dust background even for walls near the critical regime, where they start inflating.

\section{$3.2 \quad$ Initial $\mathrm{BH}$}

It would be useful to have an estimate of the black hole mass at the time of formation, $M_{\mathrm{BHi}}$, from the initial conditions. Ref. [4] gives an expression for the black hole mass formed by domain wall collapse in vacuum, $M_{\mathrm{IS}}=4 \pi \sigma R_{m}^{2}\left(1-2 \pi \sigma R_{m}\right)$, where $R_{m}$ is the maximum area radius of the wall and can be determined by eq. (3.2). Let $M_{\mathrm{BHi}}=C_{\mathrm{IS}} M_{\mathrm{IS}}$ with $C_{\mathrm{IS}}$ a coefficient. We find that solving eq. (3.2) and (3.3) gives $C_{\mathrm{IS}} \approx 1.1$ for both dust and radiation.

Now let $R_{H}=1 / H\left(t_{H}\right)$, which is simply given by $r_{i}^{3}$ for dust and $r_{i}^{2}$ for radiation. Physically, we expect $R_{m}$ to be of the same order as $R_{H}$, since the wall comes to a halt and then collapses due to its tension once it falls within the cosmological horizon. When $t_{\sigma} \gg t_{H}$, we may assume $M_{\mathrm{BHi}}=4 \pi \sigma C R_{H}^{2}$, where $C$ is a coefficient. By solving eq. (3.1), it was found in ref. [1] that $C_{\text {dust }} \approx 0.15$ and $C_{\text {radiation }} \approx 0.62$. Solving eq. (3.2) certainly gives similar results.

\subsection{Final BH}

A singularity arises soon after the apparent horizon forms. A well known drawback of the geodesic slicing that we are using is that it inevitably hits the singularity [15]. To avoid the breakdown of the simulation, we cut off the black hole region by ignoring what happens inside the apparent horizon, and use one-sided boundary conditions there. Since the apparent horizon lies inside the event horizon, it is safe to do so. Such black hole excision method [15] allows us to evolve the spacetime to a much later time and observe the mass accretion.

The evolution of the black hole mass, $M_{\mathrm{BH}}(t)$, is different for dust and radiation. It was shown in [14] that, for a dust background, all of the fluid between the apparent horizon and the exterior FRW boundary (which is also the approximate comoving location of the initial wall), gradually falls into the black hole, while the exterior region is unaffected. Eventually, we have a black hole enclosed by an empty region surrounded by a dust universe, where the final black hole mass $M_{\mathrm{BHf}}$ is simply the enclosed mass within the initial wall, and is given by $M_{H}=(1 / 2) r_{i}^{3}$ (see figure 2 ).

However, in a radiation-dominated universe, fluid keeps being pushed in and there will not be an empty layer outside the black hole horizon (see figure 3 ). The mass $M_{\mathrm{BH}}(t)$ keeps growing as the FRW energy density decreases (see figure 4). 


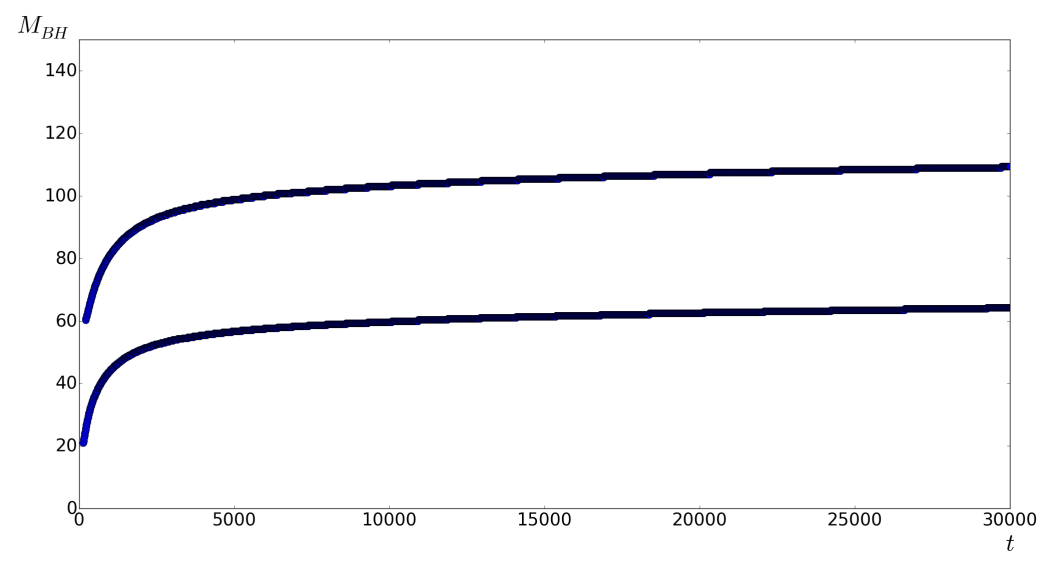

Figure 2. $M_{\mathrm{BH}}$ as a function of time for two subcritical walls in dust background with $r_{i}=5$ and 6 , and $t_{\sigma} \approx 300$. After the black hole is formed, its mass increases and converges to $M_{\mathrm{BHf}} \approx(1 / 2) r_{i}^{3}$.
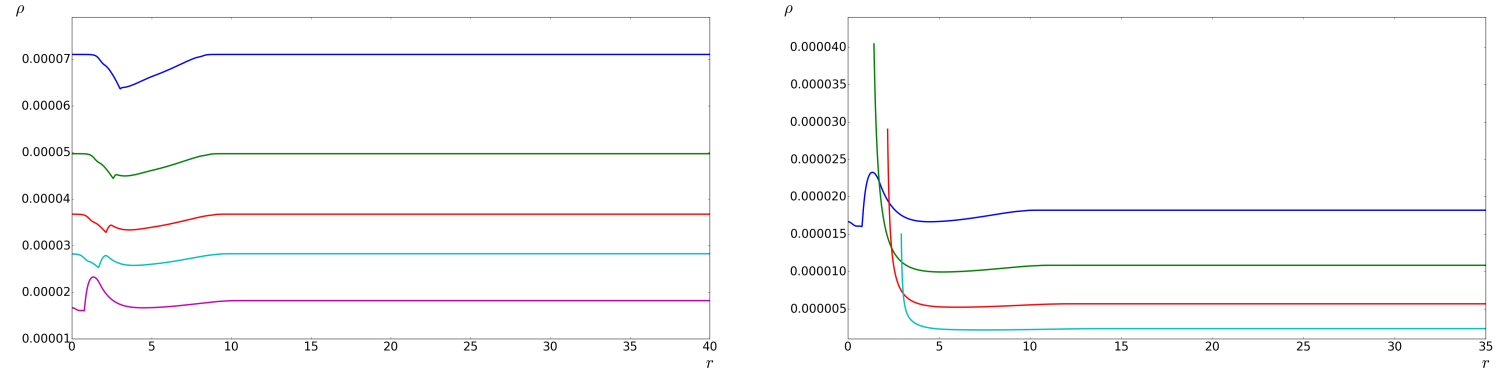

Figure 3. Evolution of the radiation energy density distribution in the case of a subcritical wall with $r_{i}=5$ and $t_{\sigma} \approx 300$. The left panel shows $\rho(r)$ at time $t=20.5,24.5,28.5,32.5,40.5$ from the top. The wall (which is not shown in the plot) is at the position where $\rho$ has the minimum value. We can see that rarefaction waves (which will be discussed later) are produced and propagate away from the wall. Meanwhile in the exterior region the energy density decreases as in an FRW universe. A "bump" develops outside the wall and will be cut off later because it will be within the black hole apparent horizon. The right panel shows $\rho(r)$ at time $t=40.5,52.5,72.5,112.5$ from the top. The black hole (which is excised for the last three moments in the plot) is not surrounded by an empty layer as it is in a dust universe. We can see that the energy deficit between the apparent horizon and the unperturbed FRW region is smoothed out with time.

The accretion of primordial black holes in the early universe was first studied in ref. [23]. If we assume that the radiation energy density right outside the black hole horizon decreases as in an FRW universe, the accretion rate of the black hole can be written as

$$
\frac{d M_{\mathrm{BH}}(t)}{d t}=4 \pi F R_{\mathrm{BH}}^{2} \rho(t)
$$

where $R_{\mathrm{BH}}=2 M_{\mathrm{BH}}$ is the Schwarzschild radius, and the accretion efficiency $F$ is expected to be a constant of order one $[24,25]$. The solution is

$$
M_{\mathrm{BH}}(t)=\frac{1}{\frac{1}{M_{0}}+\frac{3}{2} F\left(\frac{1}{t}-\frac{1}{t_{0}}\right)},
$$

where $M_{0}$ is the initial black hole mass and $t_{0}$ the time when the black hole is formed. 


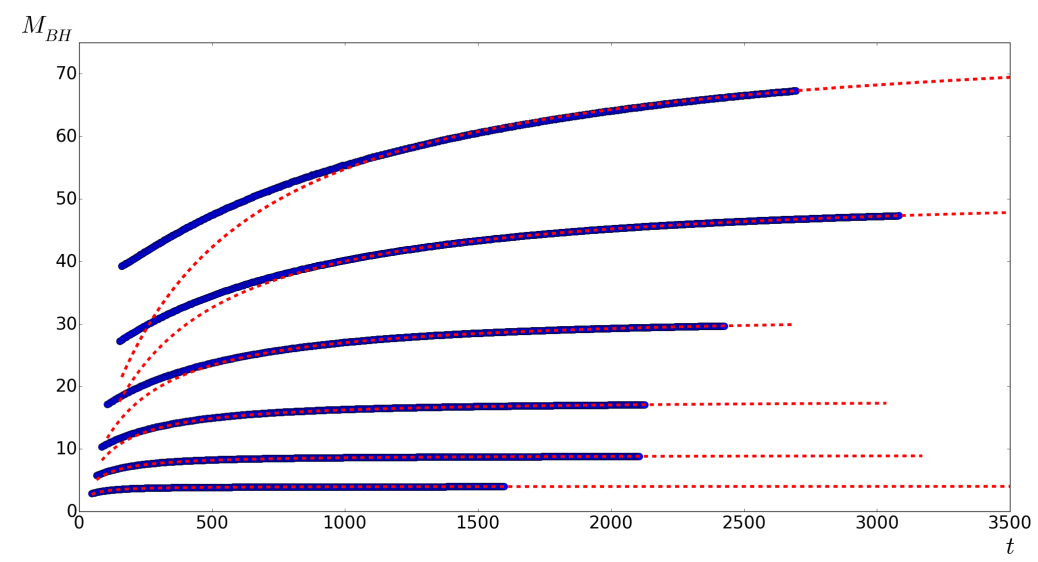

Figure 4. $M_{\mathrm{BH}}$ as a function of time for six subcritical walls in the background of radiation with the same surface tension $\left(t_{\sigma} \approx 300\right)$ but different radii. $r_{i}=5,6,7,8,9$ and 10 from the bottom. Blue curves are from simulations, and dashed red curves are from eq. (3.5). For $r_{i}=5, \frac{M_{\mathrm{BHF}}}{M_{\mathrm{BHi}}} \approx 1.5$; for $r_{i}=6, \frac{M_{\mathrm{BHf}}}{M_{\mathrm{BHi}}} \approx 1.6$; for $r_{i}=7, \frac{M_{\mathrm{BHf}}}{M_{\mathrm{BHi}}} \approx 1.8$; for $r_{i}=8, \frac{M_{\mathrm{BHf}}}{M_{\mathrm{BHi}}} \approx 1.9$; for $r_{i}=9, \frac{M_{\mathrm{BHf}}}{M_{\mathrm{BHi}}} \approx 2.0$; for $r_{i}=10$, $\frac{M_{\mathrm{BHf}}}{M_{\mathrm{BHi}}} \approx 2.0$. The ratio increases to $\sim 2$ as we approach the critical regime.

In trying to fit our numerical results with this equation, we have to take into account that the spacetime near the apparent horizon is not FRW when the black hole is formed, so $M_{0}$ is not necessarily $M_{\mathrm{BHi}}$. Instead, we choose values for $M_{0}$ and $t_{0}$ at a sufficiently late time. In the original and some subsequent papers [26, 27], $R_{\mathrm{BH}}$ is replaced by an effective capture radius of relativistic particles by the black hole, $R_{\mathrm{eff}}=(3 \sqrt{3} / 4) R_{\mathrm{BH}}$. This gives $F \sim 27 / 16$. Some argued [28] that $\rho$ should be replaced by $\rho+3 p=2 \rho$, to account for the difference between the radiation energy density and the gravitational energy density. Combining these two effects gives $F \approx 3.4$. We find that $F \approx 3.8$ works very well for the data from all our simulations. From figure 4 we can see that, for relatively large walls, the equation doesn't match the data right after the black hole formation, but works perfectly at later times. With this in mind, we don't need to run the simulation and wait until $M_{\mathrm{BH}}(t)$ converges to a constant, since as soon as eq. (3.5) is satisfied, we can immediately infer the final mass:

$$
M_{\mathrm{BHf}}=\frac{1}{\frac{1}{M_{0}}-\frac{5.7}{t_{0}}}
$$

Here $M_{0}$ and $t_{0}$ can be the values at any sufficiently late time. Our simulations suggest that the time it takes for the accretion to settle into the regime described by eq. (3.5) is about 10-100 $\mathrm{MBHi}_{\mathrm{BH}}$.

Note that for relatively small walls, the black hole doesn't grow much. By increasing $r_{i}$ or $\sigma$, the ratio $\frac{M_{\mathrm{BHf}}}{M_{\mathrm{BHi}}}$ increases until the critical regime is reached. In this regime, the ratio saturates to $\frac{M_{\mathrm{BHf}}}{M_{\mathrm{BHi}}} \approx 2$ (see captions under figure 4 and 5 ). In section 4 we will see that the size of a supercritical black hole as measured by an observer in the exterior asymptotically FRW region also doubles after its formation. 


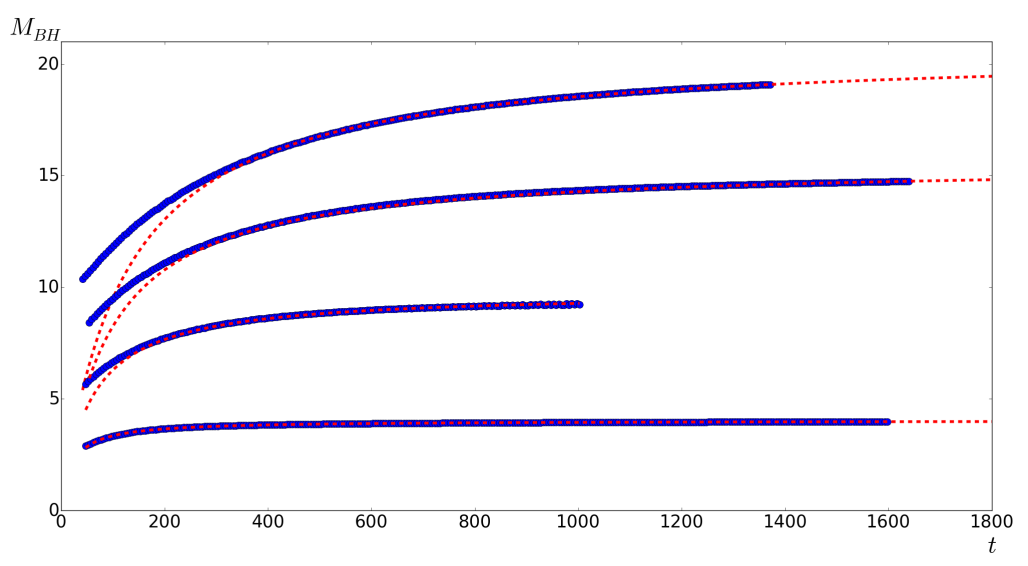

Figure 5. $M_{\mathrm{BH}}$ as a function of time for four subcritical walls in the background of radiation with the same $r_{i}(=5)$ but different surface tension. $t_{\sigma} \approx 200,100,50$ and 25 from the bottom. Blue curves are from simulations, and dashed red curves are from eq. (3.5). For $t_{\sigma}=200, \frac{M_{\mathrm{BHf}}}{M_{\mathrm{BHi}}} \approx 1.5$; for $t_{\sigma}=100$, $\frac{M_{\mathrm{BHf}}}{M_{\mathrm{BHi}}} \approx 1.8$; for $t_{\sigma}=50, \frac{M_{\mathrm{BHf}}}{M_{\mathrm{BHi}}} \approx 2.0$; for $t_{\sigma}=25, \frac{M_{\mathrm{BHf}}}{M_{\mathrm{BHi}}} \approx 2.0$. The ratio increases to $\sim 2$ as we approach the critical regime.
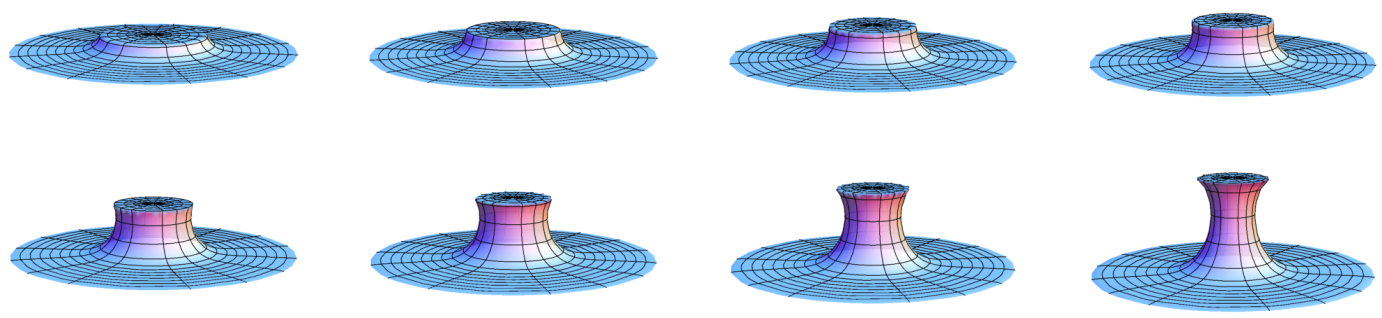

Figure 6. Embedding diagrams showing how a wormhole develops with time outside the wall. The flat-looking region at the top and the bottom area represent the interior and exterior regions respectively. The ring that encircles the top is where the wall is located. The distance from the cap center along a longitude line is the proper radius $d(r, t) \equiv \int_{0}^{r} B(\tilde{r}, t) d \tilde{r}$, while the radius of a latitude circle is the area radius $R$. The throat will be pinched off when the black hole singularity is encountered. These diagrams are not shown with the same scale. The radius of the ring should grow exponentially.

\section{Supercritical wall $\left(t_{\sigma} \lesssim t_{H}\right)$}

Gravitational collapse in the supercritical case is geometrically rather interesting. Although the wall grows without bound, it does so in a baby universe. First, a wormhole develops in the region between the wall and the exterior FRW universe. The wormhole evolves into a black hole interior region, bounded by two future black hole horizons, one facing the asymptotic FRW universe, and the other facing the baby universe (see figure 6). The wormhole eventually "pinches off" at the black hole singularity. This effectively disconnects the baby universe from the exterior parent universe.

As shown in ref. [1], if we consider a thin wall in a dust background, the wall repels the fluid nearby, and is subsequently sandwiched by two Schwarzschild layers with the same mass parameter $M_{\mathrm{BH}}$. In the strongly supercritical limit, this mass is equal to the mass of matter 


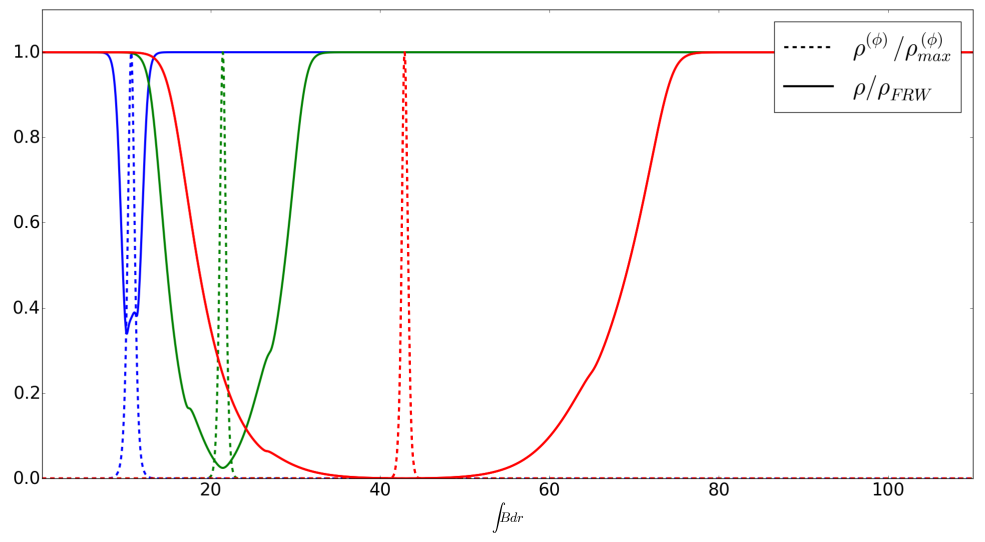

Figure 7. This graph shows how the wall pushes radiation away. Both fluid and field energy density have been rescaled so that the largest value is 1 . The blue curves are the earliest curves in this set. The $x$-axis is the proper radius $d(r, t) \equiv \int_{0}^{r} B(\tilde{r}, t) d \tilde{r}$ on a constant time hypersurface. The proper thickness of the wall barely changes.

initially enclosed by the shell. In the outer empty layer, there is a wormhole connecting two normal regions. From either side of the wormhole throat a black hole can be observed. In simulations, however, we find that the two masses $\left(M_{\mathrm{BHf}}^{(\mathrm{in})}\right.$ and $\left.M_{\mathrm{BHf}}^{(\mathrm{out})}\right)$ are not exactly identical because more fluid falls in from outside due to the initial wall thickness. More importantly, in a radiation-dominated universe, there is also mass accretion, as happened

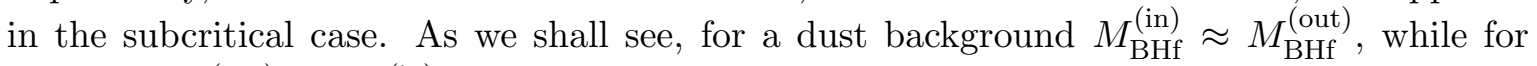
radiation $M_{\mathrm{BHf}}^{(\mathrm{out})} \approx 2 M_{\mathrm{BHf}}^{(\mathrm{in})}$.

\subsection{Spacetime structure}

Using adaptive mesh refinement, we can evolve the domain wall for some time. However, once the wall starts expanding exponentially, more and more grid points need to be introduced to cover a wall that keeps getting thinner in comoving coordinates. At that point the code becomes computationally expensive. Fortunately, in this regime the wall moves supersonically away from both the interior and the exterior regions, and becomes detached from the fluid. Afterwards, the wall remains in between two almost empty layers, where the energy density of both the scalar field and the fluid is completely negligible compared to the FRW density (figure 7). Since we are mostly interested in the black hole as it is seen from the exterior FRW region, we removed the wall and the region interior to the wall in some of the simulations. The evolution of the interior region will be considered separately in section 5 .

Let us now consider the formation and time evolution of the wormhole. Figure 8 shows the behavior of the expansion of radial null congruence as a function of radius, for different times, and figure 9 shows the spacetime structure outside the wall, with the lines separating anti-trapped from normal regions, and normal regions from trapped regions. These two graphs are obtained from a simulation of a supercritical wall in radiation background with $r_{i}=6$ and $t_{\sigma} \approx 4$. The evolution can be summarized as follows. (1) Initially, any sphere of radius bigger than the cosmological horizon $H_{i}^{-1}$ (i.e. with $r>1$ ) is anti-trapped, so $\Theta_{\text {out }}, \Theta_{\text {in }}>0$ (blue curves and slice). (2) Later, $\Theta_{\text {out }}$ reaches zero (green) at the intersection of the inner white hole horizon and the wall. Then, the slice begins to cross a normal region 


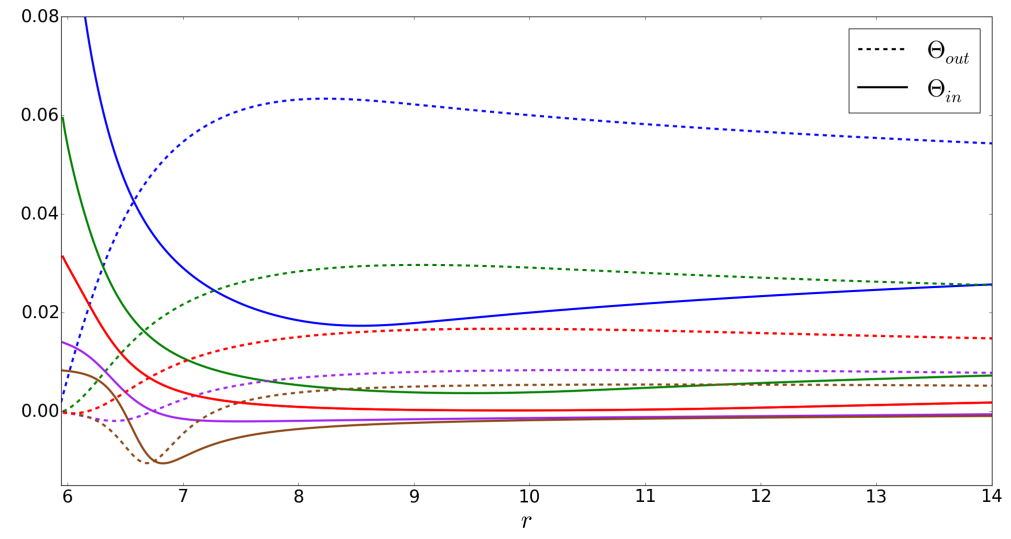

Figure 8. The evolution of the expansions outside the wall in the case of radiation background. The wall is located at $r \sim 6$. Blue curves are the earliest curves in this set. By the definitions of $\Theta_{\text {in }}$ and $\Theta_{\text {out }}$, their intersection gives the position of the wormhole throat.

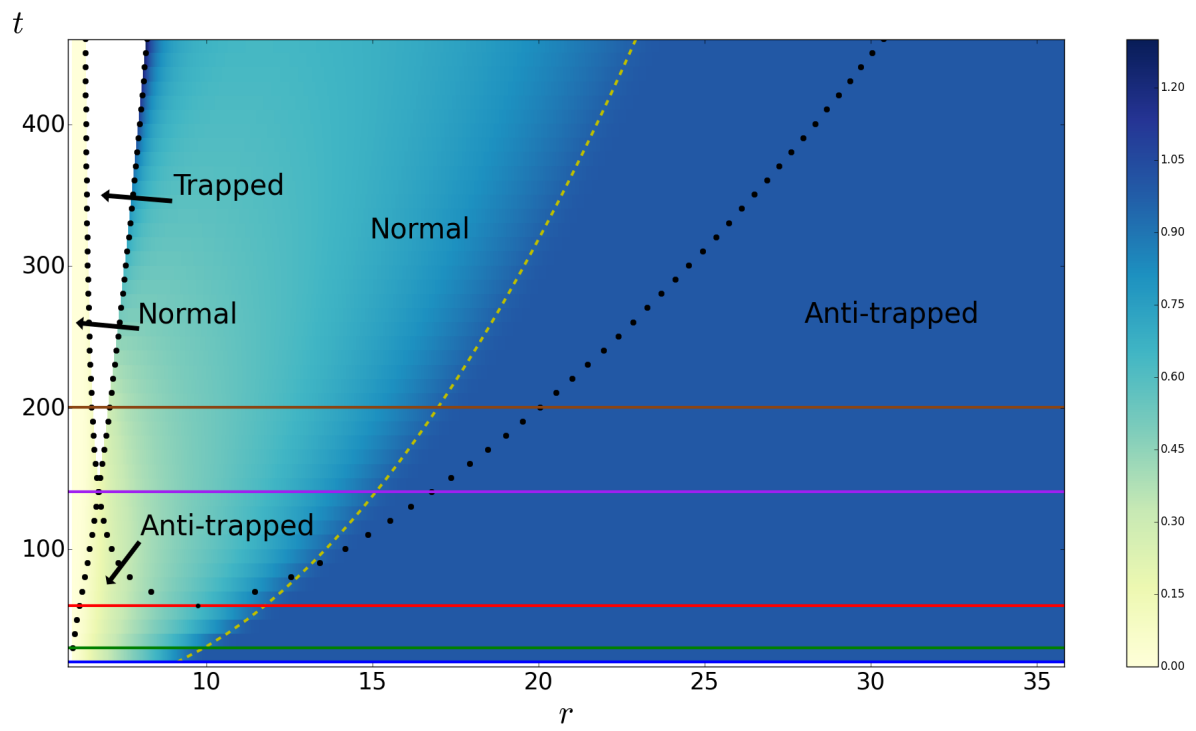

Figure 9. Depicted under simulation coordinates, this graph shows the radiation energy density distribution outside the wall. The wall is located at $r \sim 6$. The energy density has been rescaled so that $\rho_{\text {FRW }}=1$. Two apparent horizons and the cosmological horizon are shown with black dots. Five constant time slices here respectively correspond to the five different stages discussed in text. The yellow dashed curve is the rarefaction wave front trajectory (eq. (4.1)). The intersection of the yellow curve and the black dots indicates the time when the unaffected FRW region first comes within the cosmological horizon.

inside the throat, with $\Theta_{\text {out }}<0$ and $\Theta_{\text {in }}>0$ (note that this is opposite to what happens in a trivial geometry). An observer in this region would see himself inside the spherical wall and outside the wormhole. (3) Afterwards, $\Theta_{\text {in }}$ reaches zero (red), at place where the outer white hole and cosmological horizons emerge in the exterior region $(r \sim 10$ in this example). From that point on, the slice begins to cross a normal region outside the throat. (4) At a later 


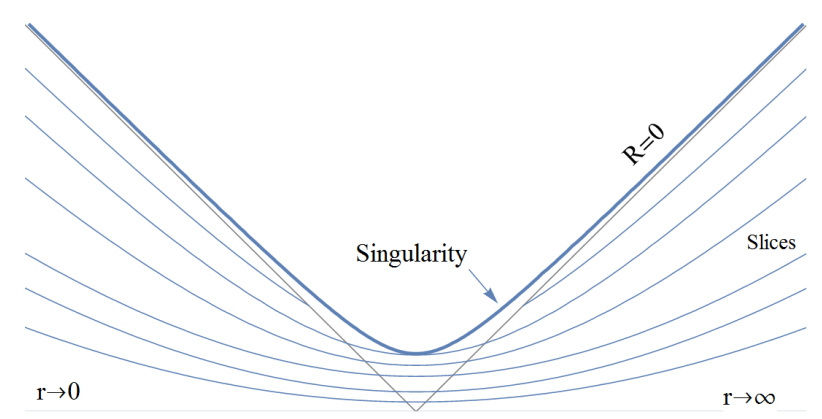

Figure 10. This sketch shows how geodesic slicing fails to avoid the singularity in Schwarzschild spacetime depicted in a Kruskal-Szekeres diagram [29]. We excise the BH region at two apparent horizons to evolve the spacetime to a much later time.

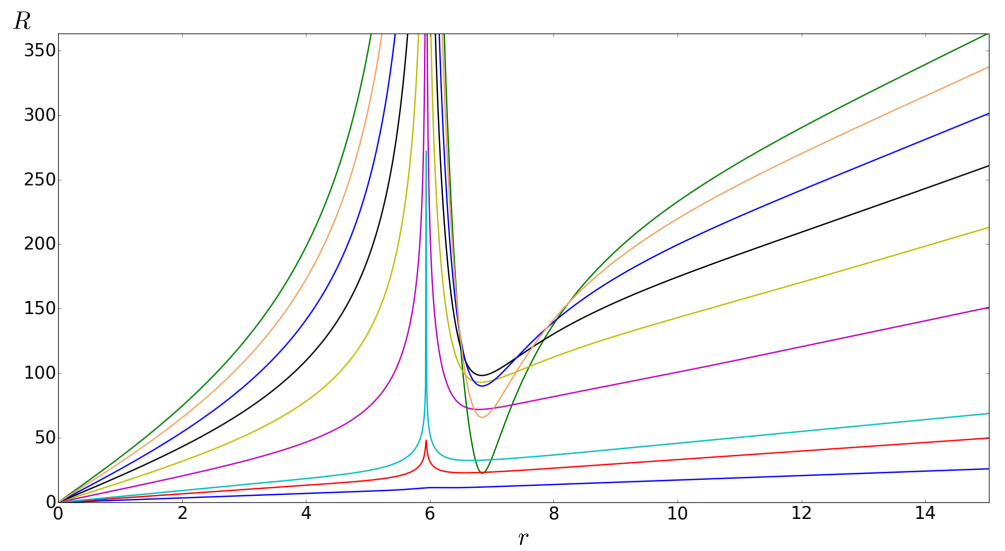

Figure 11. The area radius $R$ as a function of $r$ for a supercritical case. The bottom blue curve is the earliest curve in this set, and the green curve is the latest. Initially $R=r$, and in the unperturbed exterior region, $R=a(t) r$. Normally $R$ is monotonic in $r$, but the domain wall distorts the spacetime, so that a local minimum of $R$ develops with time, which represents the formation of a wormhole throat. The area radius of the throat increases at first, then decreases as it collapses to a black hole. A singularity is encountered when $R$ reaches zero. $R$ on the wall grows exponentially, which explains the sharp peak at $r \sim 6$, where the wall is located.

time, the marginally anti-trapped surface where $\Theta_{\text {out }}=0$ coincides with the surface where $\Theta_{\text {in }}=0$ (purple). This corresponds to a bifurcating marginal surface, and the white hole begins to turn into a black hole. (5) The slice then leaves the WH region and two apparent horizons appear with $\Theta_{\text {out }}=0\left(\Theta_{\text {in }}<0\right)$ and $\Theta_{\text {in }}=0\left(\Theta_{\text {out }}<0\right)$ respectively (brown).

As in the subcritical case, we can excise the trapped region to avoid simulation breakdown. Now we need to cut at two apparent horizons instead of one (see figure 10). However, we are still able to evolve this region for a while to see how the singularity arises. After the apparent black hole horizons form, the area radius of the throat begins to decrease and approaches zero (figures 11 and 12). Correspondingly, the fluid energy density at the throat starts to increase and eventually goes to infinity. This corresponds to the spike of growing amplitude in figure 12 . 

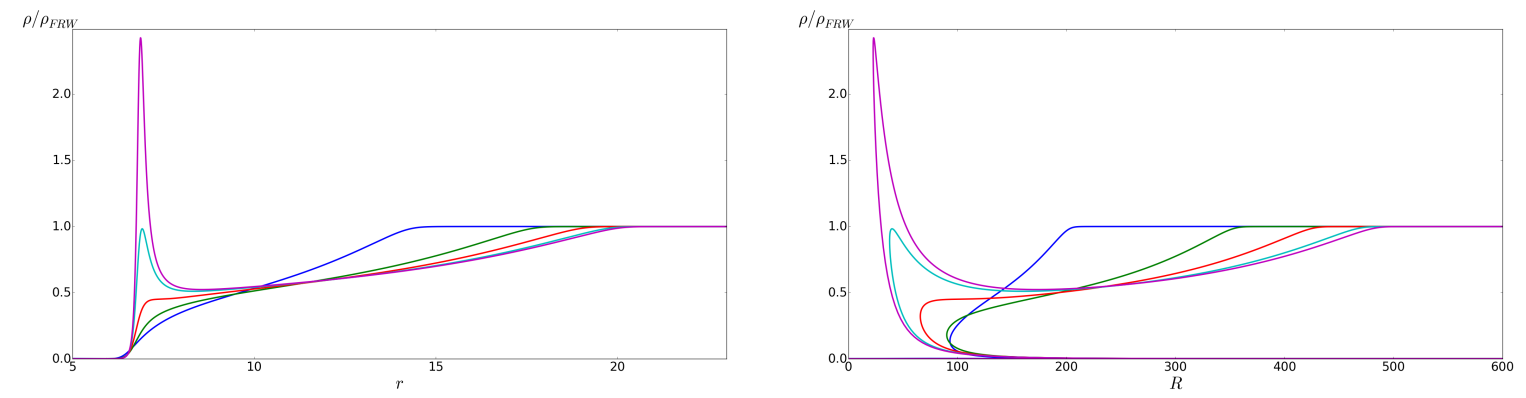

Figure 12. Evolution of the radiation energy density as the white hole turns into a black hole. The $x$-axis in the first graph is the comoving radius $r$, while in the second it is the area radius $R$. The blue curves are the earliest in this set. After apparent horizons appear, the area radius of the throat decreases and approaches zero, and fluid energy density at the throat starts to form a spike that approaches infinity, which is the singularity. $\rho(R)$ is double-valued because $R$ goes through the minimum at the throat. The upper and the lower branches correspond to the radiation energy density outside and inside the throat, respectively. The wall is located at $r \sim 6$. $\rho$ in the interior region has decreased significantly (see figure 19).

\subsection{Upper bound on the initial $\mathrm{BH}$ mass}

At the time $t_{\mathrm{BH}}$, two black hole apparent horizons with the same initial mass $M_{\mathrm{BHi}}$ are formed, at the point where $\Theta_{\text {out }}$ and $\Theta_{\text {in }}$ reach zero in the $(r, t)$ plane. Note that $M_{\mathrm{BHi}}$ should have an upper bound $\tilde{M}_{H}$, which is the total mass enclosed by the horizon crossing radius of the unperturbed FRW region. Within the cosmological horizon, a spherical surface is normal (i.e. it's not trapped nor anti-trapped). A contradiction would arise if the black hole (or white hole) apparent horizon were bigger than the cosmological horizon, because the region inside the black hole (or white hole) is trapped (or anti-trapped). Therefore, the Schwarzschild radius of the black hole cannot exceed the horizon crossing radius of the unperturbed FRW region.

In the case of dust background, if we neglect the initial wall thickness, we simply have $\tilde{M}_{H}=M_{H}=(1 / 2) r_{i}^{3}$, because the FRW boundary outside the wall is unperturbed. In the case of radiation, however, the perturbation due to the wall propagates outwards as a rarefaction (or decompression) wave. The speed of the wave is the speed of sound, $a(d r / d t)=$ $1 / \sqrt{3}$, where $a(t)=\left(t / t_{i}\right)^{1 / 2}$ and $r$ is the comoving radius of the wave front. Neglecting the wall thickness, and taking into consideration that $t_{i}=1 / 2$, the solution is

$$
r(t)=r_{i}+\frac{1}{\sqrt{3}}(\sqrt{2 t}-1)
$$

The time when this expanding wave front comes within the cosmological horizon (see figure 9) can be found from $a(t) r(t)=2 t$, which gives

$$
\tilde{M}_{H}=\tilde{t}_{H} \approx 2.8\left(r_{i}-\frac{1}{\sqrt{3}}\right)^{2}
$$

For large $r_{i}, \tilde{M}_{H} \approx 2.8 r_{i}^{2}=5.6 M_{H}$. As we shall see, the actual black hole mass is comparable to this upper bound. 


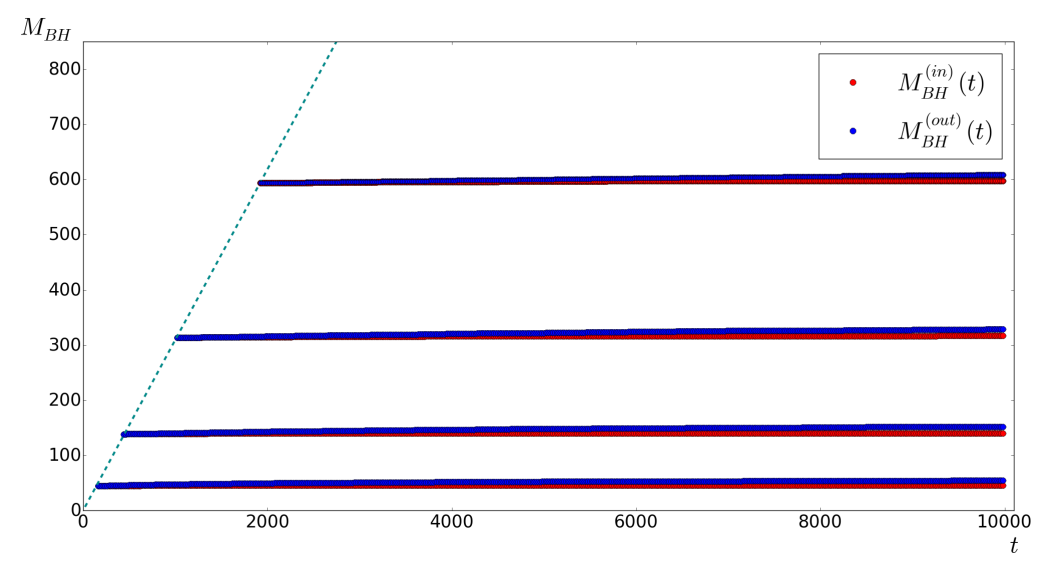

Figure 13. Evolution of $M_{\mathrm{BH}}(t)$ for four supercritical walls in the background of dust with $r_{i}=4,6,8$ and 10 from the bottom, and $t_{\sigma} \approx 3$. When the black holes are formed, the masses of the inner and the outer black hole are identical. We find that $M_{\mathrm{BHi}} \propto t_{\mathrm{BH}}$ (dashed straight line). Then $M_{\mathrm{BH}}^{(\mathrm{in})}(t)$ converges very quickly, while $M_{\mathrm{BH}}^{(\text {out })}(t)$ grows for a long time, yet doesn't increase much. The difference between the two is caused by the wall thickness. If $r_{i}$ is very large, $M_{\mathrm{BHf}}^{(\mathrm{in})} \approx M_{\mathrm{BHf}}^{(\mathrm{out})}$.

\subsection{Inner BH}

Simulations show that $M_{\mathrm{BHi}}$ and $t_{\mathrm{BH}}$ are linearly related (see figures 13 and 15). In a dustdominated universe, $M_{\mathrm{BHi}} \approx 0.31 t_{\mathrm{BH}}$; for radiation, $M_{\mathrm{BHi}} \approx 0.34 t_{\mathrm{BH}}$.

After the black holes are formed, fluid nearby falls in and the black hole masses increase. For the inner black hole the situation is not very different for dust and radiation because the energy density has a tiny value between the wall and the apparent horizon (see figure 17). Starting with $M_{\mathrm{BHi}}, M_{\mathrm{BH}}^{(\mathrm{in})}(t)$ converges very quickly (see figure 13 and 15 ), so $M_{\mathrm{BHf}}^{(\mathrm{in})} \approx M_{\mathrm{BHi}}$.

In the case of dust background, it was argued in ref. [1] that for a large supercritical wall, $M_{\mathrm{BHf}}^{(\mathrm{in})} \approx M_{H}$, where $M_{H}=(1 / 2) r_{i}^{3}$ is the enclosed mass within the initial shell. In figure 14 we can see that the ratio $M_{\mathrm{BHf}}^{(\mathrm{in})} / M_{H}$ does approach 1 with an increasing $r_{i}$. The deviation comes from the initial wall thickness and energy compensation.

As for the case of radiation, simulations show that $M_{\mathrm{BHf}}^{(\mathrm{in})} / M_{H} \approx 2.8$ as $r_{i}$ increases, where $M_{H}=(1 / 2) r_{i}^{2}$ is the enclosed mass at $t_{H}$ (see figure 16). This is about half of the upper bound estimated in eq. (4.2). As in the case of dust background, $M_{\mathrm{BHf}}^{(\mathrm{in})}$ is independent of the wall's tension.

\subsection{Outer BH}

As in the subcritical case, the evolution of $M_{\mathrm{BH}}^{(\mathrm{out})}(t)$ is very different for different fluids (figure 17). Dust between the apparent horizon and the edge of the exterior FRW region eventually falls into the black hole, while the FRW region is unaffected. If $r_{i}$ is sufficiently large, such mass accretion is negligible, and we have $M_{\mathrm{BHf}}^{(\text {out })} \approx M_{H}$ (see figure 13).

However, in the case of radiation, since fluid keeps falling in, $M_{\mathrm{BH}}^{(\mathrm{out})}(t)$ grows for a long time before it converges. We find that eq. (3.5) applies here as well at later times, again with $F \approx 3.8$. Moreover, it turns out that $M_{\mathrm{BH}}^{\text {(out) }}\left(r_{i}, t\right)$ satisfies a scaling relation (see figure 18). 


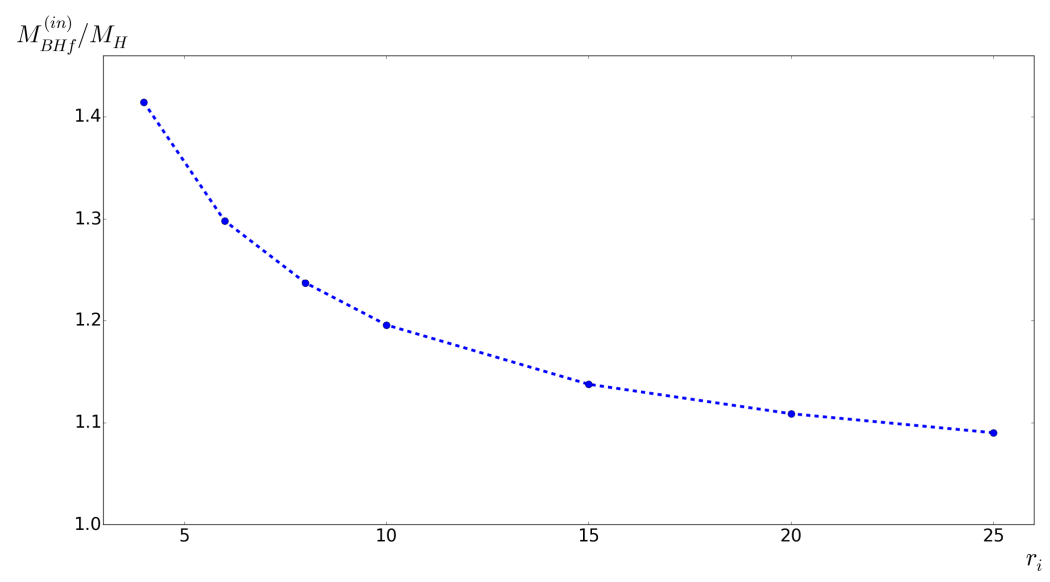

Figure 14. Blue dots are $\frac{M_{\mathrm{BHf}}^{(\mathrm{in})}}{M_{H}}$ for seven supercritical walls in the background of dust with $r_{i}=$ $4,6,8,10,15,20$ and 25 . We can see that the ratio approaches 1 as $r_{i}$ increases.

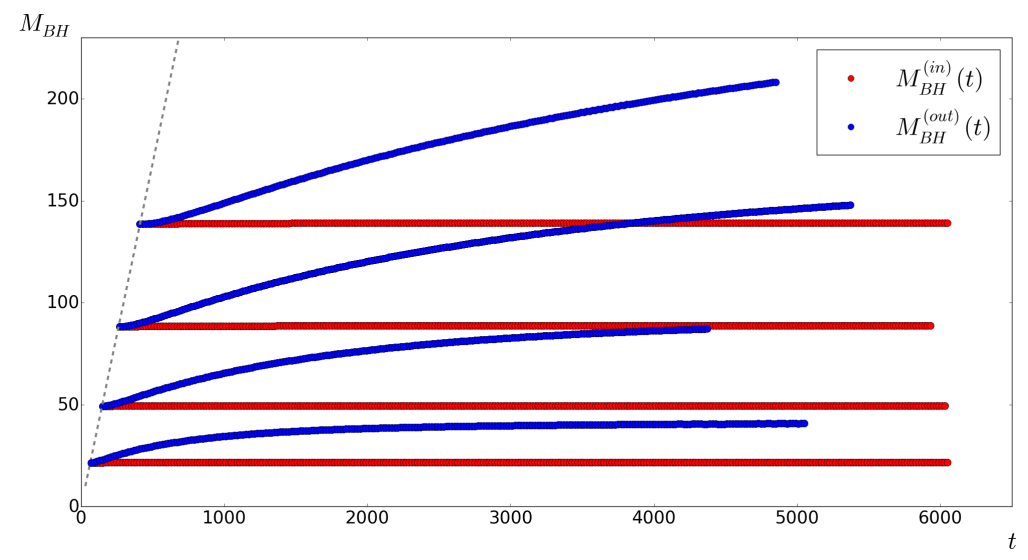

Figure 15. Evolution of $M_{\mathrm{BH}}(t)$ for four supercritical walls in the background of radiation with $r_{i}=4,6,8$ and 10 from the bottom, and $t_{\sigma} \approx 3$. Again we find that $M_{\mathrm{BHi}} \propto t_{\mathrm{BH}}$ (dashed straight line). $\quad M_{\mathrm{BH}}^{(\mathrm{in})}(t)$ converges very quickly, while $M_{\mathrm{BH}}^{(\mathrm{out})}(t)$ grows for a long time, as radiation in the exterior region keeps falling in.

For two walls labeled by 1 and 2 respectively, we find that

$$
M_{\mathrm{BH} 2}^{(\text {out })}(K t)=K M_{\mathrm{BH} 1}^{\text {(out) }}(t),
$$

where

$$
K \approx \frac{M_{\mathrm{BHi} 2}}{M_{\mathrm{BHi} 1}} \approx\left(\frac{r_{i 2}}{r_{i 1}}\right)^{2} .
$$

Since $M_{\mathrm{BH}}^{(\mathrm{out})}(t) \rightarrow M_{\mathrm{BHf}}^{(\text {out })}$ as $t \rightarrow \infty$, it follows that

$$
\frac{M_{\mathrm{BHf} 2}^{\text {(out) }}}{M_{\mathrm{BHf} 1}^{\text {(out) }}} \approx\left(\frac{r_{i 2}}{r_{i 1}}\right)^{2} .
$$

Therefore the relation between $M_{\mathrm{BHf}}^{\text {(out) }}$ and $M_{\mathrm{BHi}}$ (or $M_{H}$ ) can be determined by looking at a single case, say $r_{i}=10$. By reading $M_{0}$ and $t_{0}$ from simulations at a sufficiently late time, 


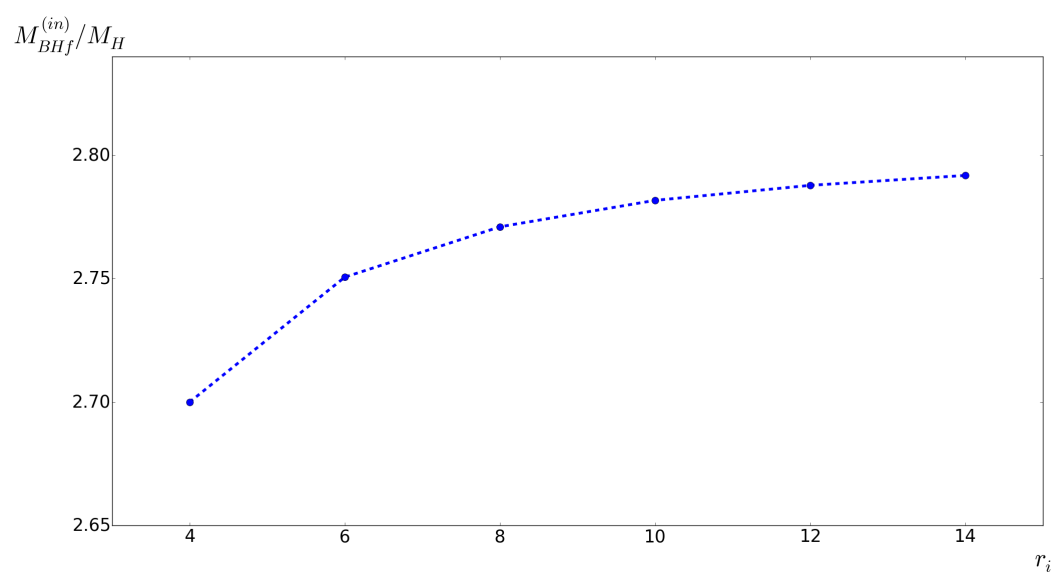

Figure 16. Blue dots are $\frac{M_{\mathrm{BHf}}^{(\mathrm{in})}}{M_{H}}$ for six supercritical walls in the background of radiation with $r_{i}=4,6,8,10,12$ and 14 . We see that the ratio approaches 2.8 as $r_{i}$ increases.

eq. (3.5) gives $M_{\mathrm{BHf}}^{\text {(out) }} \approx 276 \approx 5.5 M_{H} \approx 2 M_{\mathrm{BHi}}$. Hence we conclude that for supercritical domain walls in the background of radiation, the mass of the outer black hole roughly doubles after its formation.

\section{$5 \quad$ Fireball}

For completeness, here we consider the evolution of the interior region of a supercritical wall in radiation background. Once the empty layers are formed, as the wall inflates away, we are left with a relativistic fireball expanding into vacuum. The dynamics of this system is of interest in itself, because similar fireballs may be at the origin of $\gamma$-ray bursts (GRBs). Analytical and numerical investigations of the fireball expansion as applied to the GRB events have been done in numerous works [30-34].

Let us briefly summarize our numerical results in the present context. In the case of radiation background, a rarefaction wave is produced near the wall and then propagates towards the center, while the energy density near the center keeps decreasing as in an FRW universe. If the initial wall thickness can be neglected, the motion of the wave front is given by

$$
r(t)=r_{i}-\frac{1}{\sqrt{3}}(\sqrt{2 t}-1)
$$

where we have used that, in our units, $t_{i}=1 / 2$. When the wave reaches the center, at time

$$
t=\frac{1}{2}\left(\sqrt{3} r_{i}+1\right)^{2}
$$

the energy density near the center begins to drop much faster than that in the exterior FRW region (see figure 19). The reason is that there is no external pressure on the fireball, so it freely expands into the empty space which is opened up by the receding wall. Numerically we find $\rho(r=0, t) \propto t^{-n}$ with $n \approx 8$. Once the rarefaction wave hits the center, a reflected wave moves outwards (see figure 20). Then a leading shell is formed where most energy is concentrated and moves nearly at the speed of light (see figures 21 and 22), leaving an empty region inside the fireball. This evolution scenario is in a qualitative agreement with earlier studies of relativistic fireballs [30-34]. 

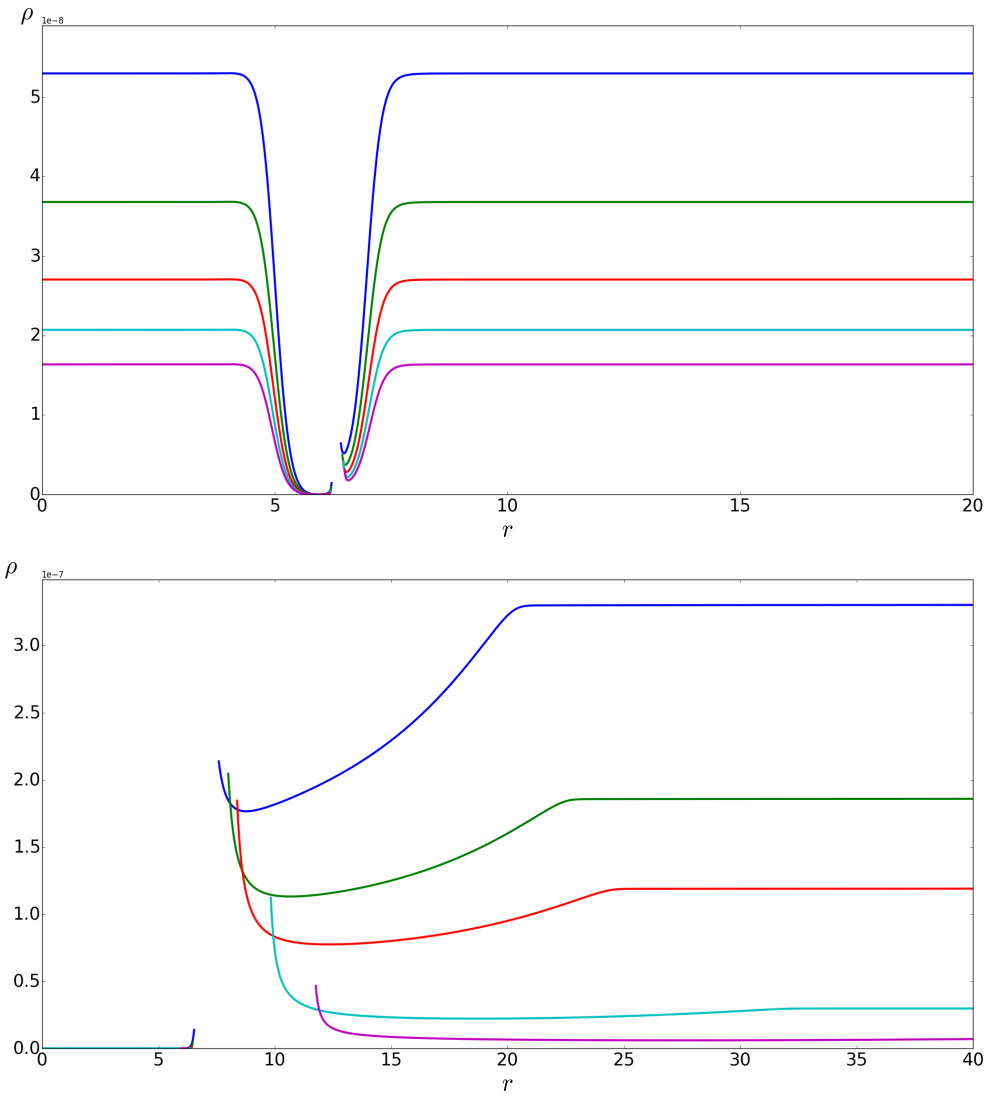

Figure 17. The upper and lower graphs illustrate the mass accretion processes for dust and radiation backgrounds, respectively. Each graph shows the energy density distributions at four different moments, with time increasing from top to bottom. The wall is located at $r \sim 6$. (To avoid simulation breakdown, we excise most of the spacetime region between the two apparent horizons.) Dust between the outer black hole horizon and the exterior FRW boundary falls in, so $M_{\mathrm{BHf}}^{\text {(out) }}$ is slightly larger than $M_{\mathrm{BHf}}^{(\mathrm{in})}$. If the wall is very large, this difference is insignificant. In the case of radiation background, the rarefaction wave propagates outwards, and the energy density approaches a uniform value. As the energy density decreases in the exterior FRW region, accretion slows down and the black hole mass converges to a constant. In radiation case, $\rho$ in the interior region has decreased significantly (see figure 19).

\section{Summary and conclusions}

In this paper we have numerically studied the gravitational collapse of spherical domain walls embedded in an FRW universe. The resulting spacetime structure depends on whether the acceleration time-scale $t_{\sigma}=(2 \pi G \sigma)^{-1}$ associated with the gravitational field of the domain wall is larger or smaller than $t_{H}\left(r_{i}\right)$. Here, $r_{i}$ is the initial radius of the wall at the time when inflation ends, and $t_{H}$ is the time at which such comoving scale would cross the horizon in an undisturbed FRW universe.

Subcritical walls are those with $t_{\sigma} \gg t_{H}$. Their gravitational field can be neglected at least up to the time $t_{H}$. At that point, they start shrinking under the pull of their own tension and eventually collapse, forming ordinary black holes. We developed a semi-analytical method to obtain the trajectory of subcritical walls, and the initial mass of the resulting black 


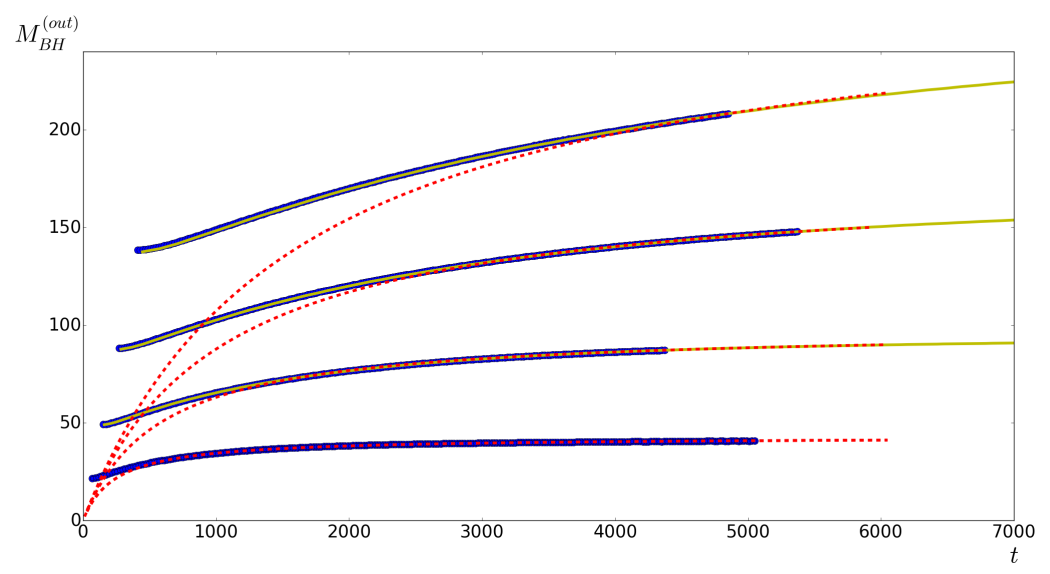

Figure 18. This graph shows $M_{\mathrm{BH}}^{(\mathrm{out})}$ as a function of time for four supercritical walls in the background of radiation with $r_{i}=4,6,8$ and 10 respectively (the same walls as those in figure 15). Blue curves are from simulations, dashed red curves from eq. (3.5), and solid yellow curves are from eq. (4.3) relative to the wall with $r_{i}=4$. For $r_{i}=6, K=1.512^{2} \approx\left(\frac{6}{4}\right)^{2}$; for $r_{i}=8, K=2.022^{2} \approx\left(\frac{8}{4}\right)^{2}$; for $r_{i}=10, K=2.533^{2} \approx\left(\frac{10}{4}\right)^{2}$.

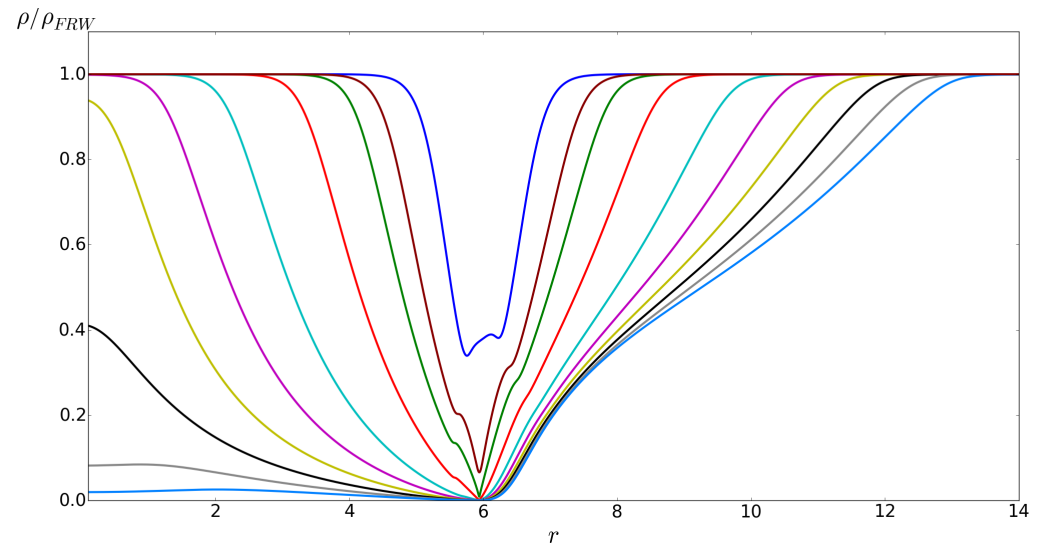

Figure 19. Propagation of the rarefaction waves. As the wave front reaches the center, the radiation energy density begins to drop much faster than that in the exterior region.

holes. For walls that collapse in a dust-dominated universe, we find that the initial mass is given by $M_{\mathrm{BHi}} \approx 5 \sigma t_{H}^{2}$, while for walls collapsing in a radiation-dominated universe we find $M_{\mathrm{BHi}} \approx 30 \sigma t_{H}^{2}$. These results are in good agreement with the numerical studies of refs. [1, 14] for the case of a matter dominated universe, and ref. [1] for the radiation-dominated case. We then turned to simulations to study the subsequent accretion of nearby fluid into the black hole.

For the matter dominated case, we find that the final mass is given by $M_{\mathrm{BHf}} \approx$ $(3 / 4) t_{H} / G$. This result is in agreement with ref. [14], and the situation can be described as follows. In our model, matter can freely pass through the domain wall, interacting with it only gravitationally. Nonetheless, all of the matter that was originally contained within the wall will either remain within the wall until the black hole forms, or it will exit the wall with less than the escape velocity, eventually falling back into the black hole. 


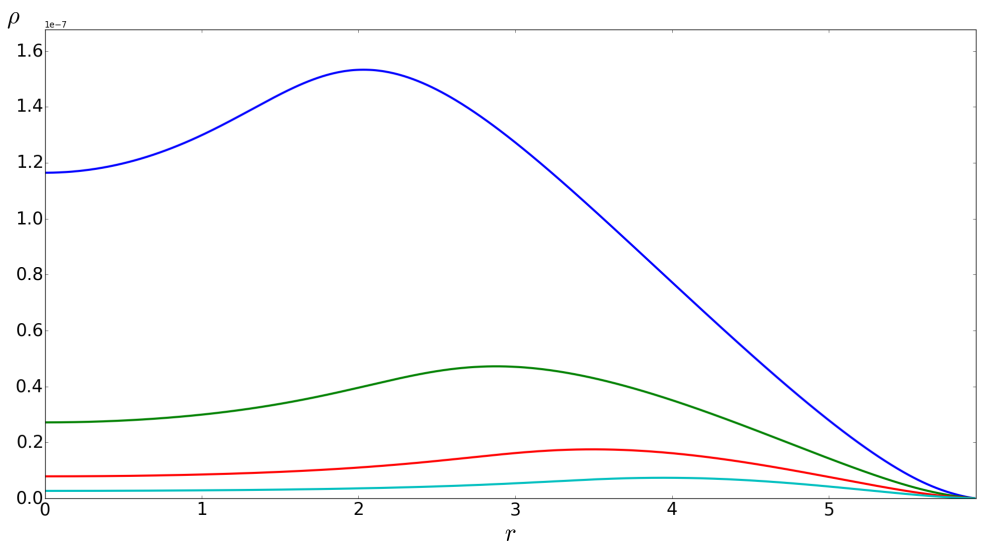

Figure 20. As the wave front reaches the center, a reflected wave is produced and begins to propagate outwards.

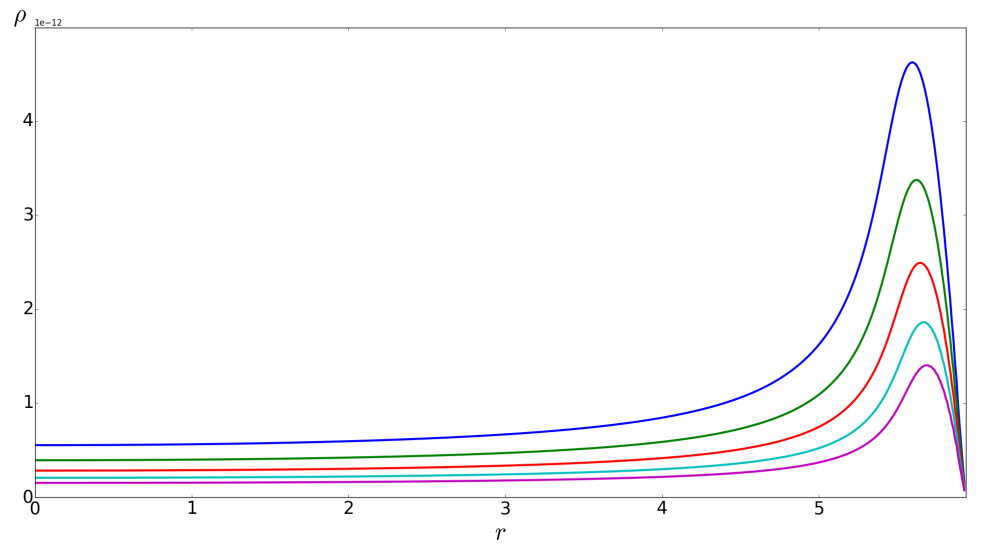

Figure 21. As the reflected wave moves outwards, a shell is formed where most energy is concentrated and moves nearly at the speed of light, leaving an empty region inside the fireball.

On the other hand, for subcritical walls in the radiation-dominated universe, mass accretion turns out to be more modest. It turns out that this is well described by Zeldovich and Novikov's model, eq. (3.4), with an accretion efficiency $F \approx 3.8$, and we find that $M_{\mathrm{BHf}} \approx \alpha M_{\mathrm{BHi}}$ where $1<\alpha<2$, and $\alpha \approx 2$ as we approach the critical limit where $t_{\sigma} \sim t_{H}$.

Supercritical walls are those with $t_{\sigma} \ll t_{H}$. At the time $t_{\sigma}$ a supercritical wall starts pushing matter away from it, while growing in size at an exponential rate. To accommodate such behavior, the wall moves through a wormhole into a baby universe, where its exponential expansion continues forever. The wormhole pinches off on a time-scale of order $t_{H}$, leaving behind two black hole horizons at the place where the wormhole mouths used to be. One of the black hole horizons faces the baby universe, while the other one faces the exterior FRW universe. At the time of formation, the interior and exterior apparent horizons coincide.

For the case of dust, the initial radius of such apparent horizons is given by $2 G M_{\mathrm{BHi}}^{(\mathrm{in})}=$ $2 G M_{\mathrm{BHi}}^{\text {(out) }} \approx(3 / 2) t_{H}$. Note that this coincides with the Hubble radius at the time $t_{H}$. Supercritical walls do not accrete any substantial amount of matter. All of the matter that 


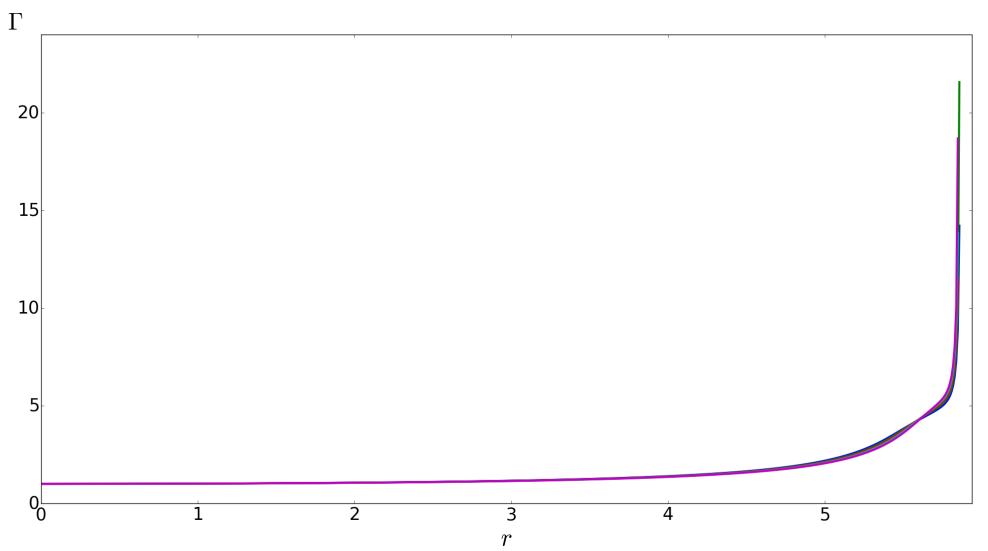

Figure 22. The Lorentz factor $\Gamma=\frac{1}{\sqrt{1-v^{2}}}$ as a function of $r$ as the shells expand in figure 21.

was initially inside the wall remains inside the wall, and never gets close to the black hole horizon which is facing the baby universe. Matter which is initially outside the wall follows the flat FRW geodesic motion, with the escape velocity relative to the black hole, so it does not accrete either. The large supercritical black holes form as a consequence of the wormhole evolution, without any matter ever crossing the black hole horizons [1].

For the case of radiation, simulations show that the initial size of the apparent horizons is given by $2 G M_{\mathrm{BHi}}^{(\mathrm{in})}=2 G M_{\mathrm{BHi}}^{\text {(out) }} \approx 5.6 t_{H} \approx \tilde{t}_{H}$. Here, $\tilde{t}_{H} \approx 3(\sqrt{3}-1)^{-2} t_{H}$ is defined as the time when the region affected by the wall motion comes within the Hubble radius. In this expression for $\tilde{t}_{H}$, we have taken into consideration that the affected comoving region grows in time, since as soon as the wall starts its exponential expansion at the time $t_{\sigma} \ll t_{H}$, a rarefaction wave propagates outward into the FRW region at the speed of sound. For instance, in the simulation represented in figure 9 , we have $\tilde{t}_{H} \approx 100 H_{i}^{-1}$. This corresponds to the time when the dotted line representing the cosmological horizon intersects the boundary of the uniform region unaffected by collapse. Note that the initial size of the apparent horizon is approximately half the size of the cosmological horizon at the time $\tilde{t}_{H}$. On the other hand, $\tilde{t}_{H}$ precedes the time $t_{\mathrm{BH}}$ when the black hole apparent horizons form, which is represented in figure 9 by a purple line. Hence, the initial size of the black holes is smaller than half of the cosmological horizon size at the time $t_{\mathrm{BH}}$.

Mass accretion does not affect the size of the inner apparent horizon $M_{\mathrm{BH}}^{(\mathrm{in})}(t)$ much, because fluid near the inner black hole has been pushed away by the wall. On the other hand, mass accretion onto the outer black hole horizon proceeds according to eq. (3.4), with the same accretion efficiency factor as in the case of subcritical walls. Furthermore, we find that $M_{\mathrm{BH}}^{(\mathrm{out})}\left(r_{i} ; t\right)$ satisfies a scaling relation, so it is enough to consider a single value of the large supercritical initial wall radius $r_{i}$. From the asymptotic behavior of this solution at large times, we find that the mass grows approximately by a factor of 2 , so that the final Schwarzschild radius is approximately given by the Hubble radius at the time $\tilde{t}_{H}$, that is, $2 G M_{\mathrm{BHf}}^{\text {(out) }} \approx 2 \tilde{t}_{H} \equiv \tilde{H}^{-1}$.

By continuity, there should be a critical state in which the wall retains a constant radius. In fact, as the critical regime is approached from the subcritical case, a wormhole throat would be formed outside the wall and a baby universe created. If the wall gravity is 


\begin{tabular}{|c|c|c|}
\hline BH mass & Dust & Radiation \\
\hline$M_{\mathrm{BHi}}$ & $\approx 5 \sigma t_{H}^{2}$ & $\approx 30 \sigma t_{H}^{2}$ \\
\hline$M_{\mathrm{BHf}}$ & $3 t_{H} / 4 G$ & $(1-2) M_{\mathrm{BHi}}$ \\
\hline
\end{tabular}

Table 1. BH masses in subcritical case.

\begin{tabular}{|c|c|c|}
\hline BH mass & Dust & Radiation \\
\hline$M_{\mathrm{BHi}}^{\text {(in) }}$ & $3 t_{H} / 4 G$ & $2.8 t_{H} / G$ \\
\hline$M_{\mathrm{BHf}}^{\text {(in) }}$ & $3 t_{H} / 4 G$ & $2.8 t_{H} / G$ \\
\hline$M_{\mathrm{BHi}}^{\text {(out) }}$ & $3 t_{H} / 4 G$ & $2.8 t_{H} / G$ \\
\hline$M_{\mathrm{BHf}}^{\text {(out) }}$ & $3 t_{H} / 4 G$ & $5.6 t_{H} / G$ \\
\hline
\end{tabular}

Table 2. BH masses in supercritical case.

still not sufficiently strong, the wall would eventually collapse, and the baby universe would disappear. However, if the wall is balanced by its tension and repulsive gravity, it would retain an asymptotically constant size. This is an unstable state, and the wall would either collapse or grow forever in the baby universe under a small perturbation.

Black hole masses in different scenarios are summarized in table 1 and table 2. Our results agree with nearly all the expectations in ref. [1], except that the supercritical black hole mass in radiation background is about 5.6 times larger than the upper bound estimated there. This corresponds to the ratio between $\tilde{t}_{H}$ and $t_{H}$. Aside from this overall factor, the black hole mass distribution obtained in ref. [1] remains unchanged.

Although the present work is motivated by the scenario where domain walls nucleate by quantum tunneling during inflation, some of our results may also be useful in scenarios where the sites of domain walls after inflation are determined by quantum diffusion of light fields during inflation $[35,36]$. This connection is worth investigating, and is left for further research. We also note that our analysis of mass accretion onto black holes may be applicable more widely, to models where primordial black holes are not formed by domain walls, but by other mechanisms (see, e.g., [37-40]).

\section{Acknowledgments}

We would like to thank Jose Juan Blanco-Pillado and Xiaozhe Hu for helpful discussions on some numerical simulation issues. This work was supported in part by the National Science Foundation under grant PHY-1518742 (H.D. and A.V.) and by MEC FPA2013-46570-C22-P, FPA2016-76005-C2-2-P, MDM-2014-0369 of ICCUB (Unidad de Excelencia "María de Maeztu"), AGAUR 2014-SGR-1474 (J.G.).

\section{A Collapse of subcritical walls}

In this appendix we use Israel's junction conditions to derive an approximate equation of motion for a subcritical domain wall. In the subcritical case, when the gravitational effect of the wall is not significant, we may assume that the interior region is a flat FRW universe with metric

$$
d s^{2}=-d t^{2}+a^{2}(t)\left(d r^{2}+r^{2} d \Omega^{2}\right) .
$$


In the exterior region the metric can be expressed as

$$
d s^{2}=-d t^{2}+a_{1}(r, t)^{2} d r^{2}+a_{2}(r, t)^{2} r^{2} d \Omega^{2} .
$$

For a thin wall, we can use the junction conditions to match metrics (A.1) and (A.2) with $a_{1}=a_{2}=a$ inside the wall. We closely follow the method and notations in [14].

Let the trajectory of the wall be $(t(\tau), r(\tau), 0,0)$, where $\tau$ is the proper time on the wall, then the tangent vector is $v^{\mu}=\left(t_{, \tau}, r_{, \tau}, 0,0\right)$, where,$\tau \equiv d / d \tau$. Assuming $\dot{t}$ to be positive, $v^{\mu} v_{\mu}=-1$ gives $t_{, \tau}=\sqrt{1+a_{1}^{2} r_{, \tau}^{2}}$. (There's an abuse of symbols here since $t$ and $r$ are used as coordinates as well as the trajectory).

Let $\xi^{\mu}$ be the normal unit vector on the wall; then $\xi^{\mu} v_{\mu}=0$ and $\xi^{\mu} \xi_{\mu}=1$ give $\xi^{\mu}=$ $\left(a_{1} r_{, \tau}, t_{, \tau} / a_{1}, 0,0\right)$ and $\xi_{\mu}=\left(-a_{1} r_{, \tau}, a_{1} t_{, \tau}, 0,0\right)$. The induced metric on the wall is $h_{\mu \nu}=$ $g_{\mu \nu}-\xi_{\mu} \xi_{\nu}$ and the extrinsic curvature is $K_{\mu \nu}=h_{\mu}{ }^{\alpha} \nabla_{\alpha} \xi_{\nu}$, where $\nabla$ is the covariant derivative operator for 4 -spacetime.

We define the brackets $[Q]_{0} \equiv Q_{\text {out }}-Q_{\text {in }}$, and $\{Q\}_{0} \equiv Q_{\text {out }}+Q_{\text {in }}$, where "out" and "in" denote matching quantities on different sides of the hypersurface. Also we use the notation $\bar{Q} \equiv\left(Q_{\text {out }}+Q_{\text {in }}\right) / 2$. Then the first junction condition is $\left[h_{\mu \nu}\right]_{0}=0$, and the second junction condition is $\left[K_{\mu \nu}\right]_{0}=8 \pi\left(-S_{\mu \nu}+S h_{\mu \nu} / 2\right)$, where $S_{\mu \nu}=-\sigma h_{\mu \nu}$ is the energy-momentum tensor of the domain wall. The equation of motion for the wall is $S_{\mu \nu} \bar{K}^{\mu \nu}=\left[T_{\mu \nu} \xi^{\mu} \xi^{\nu}\right]_{0}$. Lastly, energy conservation can be expressed as $h_{\mu}{ }^{\alpha} \nabla_{\alpha} S^{\mu}{ }_{\nu}=-\left[T_{\mu \alpha} \xi^{\mu} h^{\alpha}{ }_{\nu}\right]_{0}$.

The $(t, t)$ and $(\theta, \theta)$ components of the first junction condition give

$$
a_{1}^{2}=a_{2}^{2}=a^{2} .
$$

Therefore $a_{1, \tau}=a_{2, \tau}=a_{, \tau}$, which gives

$$
\dot{a} t_{, \tau}=a_{, \tau}=t_{, \tau} \dot{a_{1}}+r_{, \tau} a_{1}^{\prime}=t_{, \tau} \dot{a_{2}}+r_{, \tau} a_{2}^{\prime} .
$$

Furthermore, the $(\theta, \theta)$ component of the second junction condition gives

$$
\left[\xi^{\mu} \partial_{\mu} \ln \left(a_{2} r\right)\right]_{0}=-4 \pi \sigma,
$$

and the $(\tau, \tau)$ component gives

$$
\left[\xi_{\mu} D_{\tau} v^{\mu}\right]_{0}=-4 \pi \sigma
$$

where $D_{\tau} v^{\mu}=\partial_{\tau} v^{\mu}+\Gamma_{\lambda \sigma}^{\mu} v^{\lambda} v^{\sigma}$, with $\Gamma_{\lambda \sigma}^{\mu}$ the Chistoffel symbols in 4-spacetime.

A perfect fluid has energy-momentum tensor $T_{\mu \nu}=(\rho+p) u_{\mu} u_{\nu}+p g_{\mu \nu}$. Then the equation of motion is

$$
\left\{\xi_{\mu} D_{\tau} v^{\mu}+2 \xi^{\mu} \partial_{\mu} \ln \left(a_{2} r\right)\right\}_{0}=-\frac{2}{\sigma}\left[(\rho+p)\left(u^{\mu} \xi_{\mu}\right)^{2}+p\right]_{0},
$$

and the energy conservation equation takes the form

$$
\left[(\rho+p) u_{\mu} v^{\mu} u_{\nu} \xi^{\nu}\right]_{0}=0 .
$$

Let us first look at eq. (A.5). Outside the wall, we have

$$
\xi^{\mu} \partial_{\mu} \ln \left(a_{2} r\right)=a_{1} r_{, \tau} \frac{\dot{a_{2}}}{a_{2}}+\frac{t_{, \tau}}{a_{1}}\left(\frac{a_{2}^{\prime}}{a_{2}}+\frac{1}{r}\right) .
$$


Inside the wall,

$$
\xi^{\mu} \partial_{\mu} \ln (a r)=r_{, \tau} \dot{a}+\frac{t_{, \tau}}{a r}
$$

Then eq. (A.5) yields

$$
a_{2}^{\prime}=-4 \pi \sigma a^{2} \sqrt{1+a^{2} r_{, \tau}^{2}} .
$$

Similarly, manipulating eq. (A.6) gives

$$
a_{1}^{\prime}=\frac{4 \pi \sigma \sqrt{1+a^{2} r_{, \tau}^{2}}}{r_{, \tau}^{2}} .
$$

These two equations can be rewritten as

$$
\begin{aligned}
& a_{1}^{\prime}=\frac{4 \pi \sigma \sqrt{1-a^{2} \dot{r}^{2}}}{\dot{r}^{2}}, \\
& a_{2}^{\prime}=-\frac{4 \pi \sigma a^{2}}{\sqrt{1-a^{2} \dot{r}^{2}}} .
\end{aligned}
$$

Now we assume $\left[u_{\mu} \xi^{\mu}\right]_{0}=0$. Since $u_{\mu} \xi^{\mu}$ is the 4 -velocity of the fluid in the direction of the unit normal vector, this condition means the fluid flows through the wall smoothly. One then obtains

$$
\left\{\xi_{\mu} D_{\tau} v^{\mu}+2 \xi^{\mu} \partial_{\mu} \ln \left(a_{2} r\right)\right\}_{0}=0
$$

which leads to the wall's equation of motion

$$
\frac{a r_{, \tau \tau}}{\sqrt{1+a^{2} r_{, \tau}^{2}}}+\frac{4 a_{, \tau} r_{, \tau}}{\sqrt{1+a^{2} r_{, \tau}^{2}}}+\frac{2 \sqrt{1+a^{2} r_{, \tau}^{2}}}{a r}=6 \pi \sigma
$$

This can also be written as

$$
\ddot{r}+\left(4-3 a^{2} \dot{r}^{2}\right) H \dot{r}+\frac{2}{a^{2} r}\left(1-a^{2} \dot{r}^{2}\right)=6 \pi \sigma \frac{\left(1-a^{2} \dot{r}^{2}\right)^{\frac{3}{2}}}{a},
$$

which is eq. (3.2).

Furthermore, we are able to determine the time when apparent horizon arises. By eq. (2.22), when $\Theta_{\text {out }}=0$, that is, $\left(\dot{a_{2}} r\right)+\left(a_{2} r\right)^{\prime} / a_{1}=0$, one obtains

$$
\left(H+\frac{1}{a r}\right) \sqrt{\frac{1+a \dot{r}}{1-a \dot{r}}}=4 \pi \sigma
$$

which is eq. (3.3).

Lastly, the Misner-Sharp mass right outside the wall is

$$
\begin{aligned}
M & =\frac{a_{2} r}{2}\left\{1-\frac{\left(a_{2} r\right)^{\prime 2}}{a_{1}^{2}}+\left(\dot{a_{2}} r\right)^{2}\right\} \\
& =\frac{1}{2} H^{2}(a r)^{3}+\frac{4 \pi \sigma(a r)^{2}}{\sqrt{1-a^{2} \dot{r}^{2}}}+\frac{4 \pi \sigma H \dot{r}}{r \sqrt{1-a^{2} \dot{r}^{2}}}(a r)^{4}-8 \pi^{2} \sigma^{2}(a r)^{3} .
\end{aligned}
$$

These four terms are the volume energy, surface energy, surface-volume binding energy and surface-surface binding energy, respectively. When $r$ satisfies eq. (3.3), $M=a r / 2$.

If the spacetime inside the wall is Minkowski, $a=1$ and $H=0$. As a critical solution of eq. (A.17), $r \rightarrow$ const., which gives $r=\frac{1}{3 \pi \sigma}$. Then eq. (A.20) gives $M_{\mathrm{cr}}=\frac{4}{27 \pi \sigma}$, which is exactly the critical mass obtained in ref. [10]. 


\section{References}

[1] J. Garriga, A. Vilenkin and J. Zhang, Black holes and the multiverse, JCAP 02 (2016) 064 [arXiv: 1512.01819] [INSPIRE].

[2] A. Vilenkin and E.P.S. Shellard, Cosmic strings and other topological defects, Cambridge University Press, Cambridge U.K. (2000).

[3] A. Vilenkin, Gravitational field of vacuum domain walls, Phys. Lett. B 133 (1983) 177 [INSPIRE].

[4] J. Ipser and P. Sikivie, The gravitationally repulsive domain wall, Phys. Rev. D 30 (1984) 712 [INSPIRE].

[5] A. Lazanu, C.J.A.P. Martins and E.P.S. Shellard, Contribution of domain wall networks to the CMB power spectrum, Phys. Lett. B $\mathbf{7 4 7}$ (2015) 426 [arXiv: 1505. 03673] [InSPIRE].

[6] R. Basu, A.H. Guth and A. Vilenkin, Quantum creation of topological defects during inflation, Phys. Rev. D 44 (1991) 340 [InSPIRE].

[7] K. Sato, M. Sasaki, H. Kodama and K.-i. Maeda, Creation of wormholes by first order phase transition of a vacuum in the early universe, Prog. Theor. Phys. 65 (1981) 1443 [InSPIRE].

[8] H. Kodama, M. Sasaki, K. Sato and K.-i. Maeda, Fate of wormholes created by first order phase transition in the early universe, Prog. Theor. Phys. 66 (1981) 2052 [InSPIRE].

[9] V.A. Berezin, V.A. Kuzmin and I.I. Tkachev, Thin wall vacuum domains evolution, Phys. Lett. B 120 (1983) 91 [inSPIRE].

[10] S.K. Blau, E.I. Guendelman and A.H. Guth, The dynamics of false vacuum bubbles, Phys. Rev. D 35 (1987) 1747 [INSPIRE].

[11] T. Harada and B.J. Carr, Super-horizon primordial black holes, Phys. Rev. D 72 (2005) 044021 [astro-ph/0508122] [INSPIRE].

[12] H. Maeda, T. Harada and B.J. Carr, Cosmological wormholes, Phys. Rev. D 79 (2009) 044034 [arXiv:0901.1153] [INSPIRE].

[13] B.J. Carr and T. Harada, Separate universe problem: 40 years on, Phys. Rev. D 91 (2015) 084048 [arXiv: 1405.3624] [INSPIRE].

[14] N. Tanahashi and C.-M. Yoo, Spherical domain wall collapse in a dust universe, Class. Quant. Grav. 32 (2015) 155003 [arXiv:1411.7479] [INSPIRE].

[15] T.W. Baumgarte and S.L. Shapiro, Numerical relativity: solving Einstein's equations on the computer, Cambridge University Press, Cambridge U.K. (2010).

[16] I. Cho and A. Vilenkin, Space-time structure of an inflating global monopole, Phys. Rev. D 56 (1997) 7621 [gr-qc/9708005] [INSPIRE].

[17] J. Bloomfield, D. Bulhosa and S. Face, Formalism for primordial black hole formation in spherical symmetry, arXiv:1504.02071 [INSPIRE].

[18] M.J. Berger and J. Oliger, Adaptive mesh refinement for hyperbolic partial differential equations, J. Comput. Phys. 53 (1984) 484 [INSPIRE].

[19] C.L. Wainwright et al., Simulating the universe(s): from cosmic bubble collisions to cosmological observables with numerical relativity, JCAP 03 (2014) 030 [arXiv:1312.1357] [INSPIRE].

[20] M.K. Bowen and R. Smith, Derivative formulae and errors for non-uniformly spaced points, Proc. Roy. Soc. Lond. A 461 (2005) 1975.

[21] C.W. Misner and D.H. Sharp, Relativistic equations for adiabatic, spherically symmetric gravitational collapse, Phys. Rev. 136 (1964) B571 [InSPIRE]. 
[22] S.A. Hayward, Gravitational energy in spherical symmetry, Phys. Rev. D 53 (1996) 1938 [gr-qc/9408002] [INSPIRE].

[23] Y.B. Zel'dovich and I.D. Novikov, The hypothesis of cores retarded during expansion and the hot cosmological model, Astron. Zh. 43 (1966) 758.

[24] R. Guedens, D. Clancy and A.R. Liddle, Primordial black holes in brane world cosmologies: accretion after formation, Phys. Rev. D 66 (2002) 083509 [astro-ph/0208299] [INSPIRE].

[25] B. Nayak and L.P. Singh, Accretion, primordial black holes and standard cosmology, Pramana 76 (2011) 173.

[26] E.V. Bugaev, M.G. Elbakidze and K.V. Konishchev, Baryon asymmetry of the universe from evaporation of primordial black holes, Phys. Atom. Nucl. 66 (2003) 476 [Yad. Fiz. 66 (2003) 504] [astro-ph/0110660] [INSPIRE].

[27] A.S. Majumdar, Domination of black hole accretion in brane cosmology, Phys. Rev. Lett. 90 (2003) 031303 [astro-ph/0208048] [INSPIRE].

[28] P.S. Custodio and J.E. Horvath, Evolution of a primordial black hole population, Phys. Rev. D 58 (1998) 023504 [astro-ph/9802362] [INSPIRE].

[29] E. Gourgoulhon, 3+1 formalism in general relativity: bases of numerical relativity, Springer Science \& Business Media, Vol. 846 (2012).

[30] J. Goodman, Are gamma-ray bursts optically thick?, Astrophys. J. 308 (1986) L47 [INSPIRE].

[31] B. Paczynski, Gamma-ray bursters at cosmological distances, Astrophys. J. 308 (1986) L43 [INSPIRE].

[32] T. Piran, A. Shemi and R. Narayan, Hydrodynamics of relativistic fireballs, Mon. Not. Roy. Astron. Soc. 263 (1993) 861 [astro-ph/9301004] [INSPIRE].

[33] G.S. Bisnovatyi-Kogan and M.V.A. Murzina, Early stages of relativistic fireball expansion, Phys. Rev. D 52 (1995) 4380 [INSPIRE].

[34] S. Kobayashi, T. Piran and R. Sari, Hydrodynamics of a relativistic fireball: the complete evolution, Astrophys. J. 513 (1999) 669 [astro-ph/9803217] [INSPIRE].

[35] S.G. Rubin, M.Y. Khlopov and A.S. Sakharov, Primordial black holes from nonequilibrium second order phase transition, Grav. Cosmol. 6 (2000) 51 [hep-ph/0005271] [INSPIRE].

[36] M.Y. Khlopov, S.G. Rubin and A.S. Sakharov, Primordial structure of massive black hole clusters, Astropart. Phys. 23 (2005) 265 [astro-ph/0401532] [INSPIRE].

[37] B.J. Carr, Primordial black holes: do they exist and are they useful?, in Inflating horizons in particle astrophysics and cosmology, H. Susuki et al. eds., Universal Academy Press, Tokyo Japan (2005), pp. 119-149 [astro-ph/0511743] [InSPIRE].

[38] M.Y. Khlopov, Primordial black holes, Res. Astron. Astrophys. 10 (2010) 495 [arXiv:0801.0116] [INSPIRE].

[39] P.H. Frampton, M. Kawasaki, F. Takahashi and T.T. Yanagida, Primordial black holes as all dark matter, JCAP 04 (2010) 023 [arXiv: 1001.2308] [INSPIRE].

[40] S. Clesse and J. García-Bellido, Massive primordial black holes from hybrid inflation as dark matter and the seeds of galaxies, Phys. Rev. D 92 (2015) 023524 [arXiv:1501.07565] [INSPIRE]. 